\title{
Constant Round Maliciously Secure 2PC with Function-independent Preprocessing using LEGO
}

\author{
Jesper Buus Nielsen \\ Aarhus University \\ jbn@cs.au.dk
}

\author{
Thomas Schneider \\ Technische Universität Darmstadt \\ thomas.schneider@crisp-da.de
}

\author{
Roberto Trifiletti \\ Aarhus University \\ roberto@cs.au.dk
}

\begin{abstract}
Secure two-party computation (S2PC) allows two parties to compute a function on their joint inputs while leaking only the output of the function. At TCC 2009 Orlandi and Nielsen proposed the LEGO protocol for maliciously secure $2 \mathrm{PC}$ based on cut-and-choose of Yao's garbled circuits at the gate level and showed that this is asymptotically more efficient than on the circuit level. Since then the LEGO approach has been improved upon in several theoretical works, but never implemented. In this paper we describe further concrete improvements and provide the first implementation of a protocol from the LEGO family. Our protocol has a constant number of rounds and is optimized for the offline/online setting with function-independent preprocessing. We have benchmarked our prototype and find that our protocol can compete with all existing implementations and that it is often more efficient. As an example, in a LAN setting we can evaluate an AES-128 circuit with online latency down to $1.13 \mathrm{~ms}$, while if evaluating 128 AES-128 circuits in parallel the amortized cost is $0.09 \mathrm{~ms}$ per AES-128. This online performance does not come at the price of offline inefficiency as we achieve comparable performance to previous, less general protocols, and significantly better if we ignore the cost of the function-independent preprocessing. Also, as our protocol has an optimal 2-round online phase it is significantly more efficient than previous protocols when considering a high latency network.
\end{abstract}

Keywords-Secure Two-party Computation, Implementation, LEGO, XOR-Homomorphic Commitments, Selective OT-Attack

\section{INTRODUCTION}

Secure two-party computation is the area of cryptology dealing with two mutually distrusting parties wishing to compute an arbitrary function $f$ on private inputs. Say $\mathrm{A}$ has

\footnotetext{
We acknowledge support from the Danish National Research Foundation and The National Science Foundation of China (under grant 61361136003) for the Sino-Danish Center for the Theory of Interactive Computation and from the Center for Research in Foundations of Electronic Markets (CFEM), supported by the Danish Strategic Research Council. Also partially supported by the European Research Commission Starting Grant 279447.

This work was partially funded by the German Federal Ministry of Education and Research (BMBF) within CRISP, by the DFG as part of project E3 within CRC 1119 CROSSING, and by the European Union's 7th Framework Program (FP7/2007-2013) under grant agreement n. 609611 (PRACTICE).
}

Permission to freely reproduce all or part of this paper for noncommercial purposes is granted provided that copies bear this notice and the full citation on the first page. Reproduction for commercial purposes is strictly prohibited without the prior written consent of the Internet Society, the first-named author (for reproduction of an entire paper only), and the author's employer if the paper was prepared within the scope of employment.

NDSS '17, 26 February - 1 March 2017, San Diego, CA, USA

Copyright 2017 Internet Society, ISBN 1-1891562-46-0

http://dx.doi.org/10.14722/ndss.2017.23075 input $x$ and $\mathrm{B}$ has input $y$. The guarantee offered from securely computing $f$ is that the only thing learned from the computation is the output $z=f(x, y)$, in particular nothing is revealed about the other party's input that cannot be inferred from the output $z$. This seemingly simple guarantee turns out to be extremely powerful and several real-world applications and companies using secure computation have arisen in recent years [1]-[3], [15], [16]. The idea of secure computation was initially conceived in 1982 by Andrew Yao [70], [71], particularly for the semi-honest setting, in which all parties are assumed to follow the protocol specification but can try to extract as much information as possible from the protocol execution. Yao gave an approach for preventing any such extraction using a technique referred to as the garbled circuit technique. At a very high level, using the abstraction of [12], A starts by garbling or "encrypting" the circuit $f$ using the garbling algorithm $(F, e, d)=\mathrm{Gb}(f)$ obtaining a garbled circuit $F$, an input encoding function $e$ and an output decoding function $d$. It then encodes its input as $X=\operatorname{En}(x, e)$ and sends $(F, X, d)$ to $\mathrm{B}$. Then, using oblivious transfer (OT), A blindly transfers a garbled version $Y$ of B's input which enables B to compute a garbled output $Z=\operatorname{Ev}(F, X \| Y)$ which it can then decode to obtain $z=\operatorname{De}(Z, d)$. Finally $\mathrm{B}$ returns $z$ to $\mathrm{A}$.

As already mentioned, the above sketched approach can be proven secure in the semi-honest setting [52]. In the stronger malicious setting however it completely breaks down as in this model the parties are allowed to deviate arbitrarily from the protocol specification. One of the most obvious attacks is for $\mathrm{A}$ to garble a different function $f^{\prime} \neq f$ which could enable $\mathrm{A}$ to learn $y$ from the resulting value $z^{\prime}$ without $\mathrm{B}$ even noticing this. To combat this type of attack the cut-and-choose technique can be applied: instead of garbling a single circuit, $\mathrm{A}$ garbles several copies and sends these to $B$. A random subset of them is now selected for checking by $B$ and if everything is correct, some fraction of the remaining circuits must be correct with overwhelming probability. Leaving out many details these can now be evaluated to obtain a single final output. While this approach thwarts the above attack it unfortunately opens up for several new ones that also need to be dealt with, for instance ensuring input consistency for all the remaining evaluation garbled circuits. Another more subtle issue in the malicious setting is the Selective-OT Attack (also called Selective Failure Attack) as pointed out in [46], [57]. A corrupt $A$ can cheat when offering $B$ the garbled inputs in the OT step by using a bogus value for either the 0 or the 1 input. This will either result in B aborting as it cannot evaluate the garbled circuit or it will go through undetected and $B$ will return the output to $A$. Either way the input bit of $B$ is revealed to $A$ which is a 


\begin{tabular}{lccc|cccc}
\hline & \multicolumn{3}{c}{ Secret Sharing-based } & \multicolumn{3}{c}{ Garbled Circuit-based } \\
\hline Protocol & {$[45]^{*}$} & {$[28]^{*}$} & {$[26]^{*}$} & {$[69]$} & {$[56]$} & {$[65]$} & This Work \\
Constant round & $\boldsymbol{x}$ & $\boldsymbol{x}$ & $\boldsymbol{x}$ & $\checkmark$ & $\checkmark$ & $\checkmark$ & $\checkmark$ \\
Independent preprocessing & $\mathrm{N} / \mathrm{A}$ & $\boldsymbol{x}$ & $\boldsymbol{x}$ & $\boldsymbol{x}$ & $\boldsymbol{x}$ & $\boldsymbol{x}$ & $\mathbf{1 3 . 8 4} \mathbf{~ m s}$ \\
Dependent preprocessing & $\boldsymbol{x}$ & $\mathrm{N} / \mathrm{A}$ & $\mathrm{N} / \mathrm{A}$ & $62 \mathrm{~ms}$ & $74 \mathrm{~ms}$ & $5.1 \mathrm{~ms}$ & $\mathbf{0 . 7 4} \mathbf{~ m s}$ \\
Best online latency & $12 \mathrm{~ms}$ & $6 \mathrm{~ms}$ & $\mathbf{1 . 0 5} \mathbf{m s}$ & $21 \mathrm{~ms}$ & $7 \mathrm{~ms}$ & $1.3 \mathrm{~ms}$ & $1.13 \mathrm{~ms}$ \\
Best online throughput & $\sim 1 \mathrm{~ms}$ & $0.4 \mathrm{~ms}$ & $\mathbf{0 . 4 5} \boldsymbol{\mu s}$ & N/A & N/A & $0.26 \mathrm{~ms}$ & $0.08 \mathrm{~ms}$ \\
\hline
\end{tabular}

TABLE I. OVERVIEW OF STATE-OF-THE-ART PROTOCOLS AND THE BEST REPORTED TIMINGS FOR SECURELY EVALUATING AES-128 WITH MALICIOUS SECURITY ON A LAN IN THE OFFLINE/ONLINE SETTING. ALL TIMINGS ARE PER AES-128. N/A STANDS fOR Not AVAILABLE.

direct breach of security. It is easy to see that using this attack A can learn any $l$ bits of B's input with probability $2^{-l}$ if not properly dealt with.

Over the last decade several solutions to the above issues have been proposed, along with dramatic efficiency improvements for secure $2 \mathrm{PC}$ protocols based on the cut-and-choose approach of garbled circuits [4], [18], [32], [36], [39], [40], [42], [48], [50], [51], [53], [58], [64], [66], [67], [69]. Finally we note for completeness that secure computation has also been studied in great detail for many other settings, including the more general multi-party case (MPC). Several different adversarial models such as honest majority [14], [22], [37], dishonest majority [27] and covert security [7] have also been proposed in the literature. In this work we focus solely on the special case of two parties with malicious security and in the next section we discuss the reported concrete efficiency of state-of-the-art protocols in this setting.

\section{A. Related Work}

In the less than 10 years since the first reported implementation of maliciously secure 2PC based on garbled circuits [54], the performance advancements have been enormous [4], [32], [36], [48], [56], [64]-[67], [69]. Furthermore different settings and hardware configurations have been explored, notably using commodity grade GPUs in [32], [36] and large-scale CPU clusters [67] to parallelize the bulk of the computation. In the single-execution setting based solely on standard hardware the best reported performance time is that of [69] which evaluates an AES-128 circuit in total time $65 \mathrm{~ms}$. In addition, the works of [56] and [65] explore the more restricted setting of amortizing secure 2PC based on the cut-and-choose and the dual execution approach, respectively. By amortizing we mean that the protocols exploit constructing multiple secure evaluations of the same function $f$ yielding impressive performance benefits over the more general single execution setting. Furthermore these protocols are in the offline/online setting where the bulk of the computation and communication can be done before the inputs are determined. We highlight that for both protocols, the offline computation depends on the function to be computed and we will refer to this as dependent preprocessing. However both protocols allow for the inputs to be chosen sequentially when securely evaluating $f$. This allows for a low latency online phase which is desirable for many applications. For securely computing 1024 AES-128 circuits, [56] reports $74 \mathrm{~ms}$ offline and $7 \mathrm{~ms}$ online per evaluation, while the more recent [65] reports $5.1 \mathrm{~ms}$ offline and $1.3 \mathrm{~ms}$ online for the same setting. Furthermore [65] achieves a $0.26 \mathrm{~ms}$ online phase when considering throughput alone, i.e. batched evaluation.
Another direction in secure computation is the secret sharing approach where the parties initially secret share their inputs and then interactively compute the function $f$ in a secure manner. A particularly nice property of these protocols is that when considering the offline/online setting the offline phase can usually be done independently of the circuit $f$ which we call independent preprocessing. This allows for naively utilizing parallelism in the preprocessing phase and also adds more flexibility as the offline material produced is universal. Another benefit is that in general this secretsharing technique works for any number of parties and over any field, which depending on the desired functionality $f$ can significantly increase performance. We note however that these protocols usually employ expensive public-key cryptography in the preprocessing phase and are therefore much slower than the offline phases of e.g. [56], [65]. Finally the inherent interactiveness of the online phase, which has $\mathcal{O}(\operatorname{depth}(f))$ rounds of interaction, makes these protocols ill-suited for high latency networks such as WANs. There are many variations of the secret sharing approach but they typically enjoy the same overall pros and cons in terms of independent preprocessing and required interactivity. Examples of recent protocols following this paradigm are: TinyOT [19], [49], [60], SPDZ [24], [27], [44], [45], MiniMac [25], [28], [29], and TinyTables [26]. The fastest reported online time for computing an AES-128 circuit in this setting is $1.05 \mathrm{~ms}$ by [26] using dependent preprocessing. The work of [45] reports $12 \mathrm{~ms}$ online time using independent preprocessing, but the evaluation exploits the algebraic structure of AES-128. Furthermore [26] has an impressive throughput of $0.45 \mu$ s per AES-128 while [45] and [28] have throughput $\sim 1 \mathrm{~ms}$ and $0.4 \mathrm{~ms}$, respectively.

In Table I we give an overview of the properties of the mentioned protocols and the reported timings for securely evaluating AES-128 on LAN in the offline/online setting. As the secret sharing-based, non-constant round, protocols are illsuited for high latency networks we omit this from Table I since no AES-128 timings are published for these schemes in a WAN setting (however see Section VI-B for a WAN comparison of the garbled circuit protocols). The timings reported for [26], [56], [65] and This Work are all measured on the same hardware (Amazon Web Services, c4.8xlarge instances on 10 Gbit LAN), while the timings for [69] are on a less powerful instance (Amazon Web Services, c4.2xlarge instances on 2.5 Gbit LAN). Finally the results of [28], [45] have been obtained on high-end Desktop machines with $1 \mathrm{Gbit}$ LAN. The timings of [56], [65] and This Work are all for 1024 AES-128 evaluations, while those of [69] are for a singleexecution. We believe the difference in performance between the offline/online $(62 \mathrm{~ms}+21 \mathrm{~ms})$ and total latency $(65 \mathrm{~ms})$ 
settings for [69] can be explained by the inability to interleave the sending/checking and evaluation of garbled circuits in the offline/online setting. In summary, as can be seen in the table our work is the first implementation of a protocol combining the advantages of independent (and dependent) preprocessing using only a constant number of rounds.

\section{B. $L E G O$}

The Large Efficient Garbled-circuit Optimization (LEGO) was first introduced by Nielsen and Orlandi in [61] which showed a new approach for maliciously secure 2PC based on cut-and-choose of garbled gates. This gave an asymptotic complexity improvement to $\mathcal{O}(s / \log (|f|))$ as opposed to $\mathcal{O}(s)$ for the standard circuit cut-and-choose approach for statistical security $s$. However the construction of [61] was heavily based on expensive public-key cryptography and was mainly considered an asymptotic advancement. This was later improved in the two follow-up works of MiniLEGO [35] and TinyLEGO [33] yielding incrementing asymptotic and concrete efficiency improvements. In a nutshell, the LEGO technique works by the generator A first garbling multiple individual AND gates (as opposed to garbling entire circuits) and sending these to the evaluator $\mathrm{B}$. Then a cut-and-choose step on a random subset of these gates is carried out and finally the remaining unchecked gates are combined (or soldered) into a garbled fault tolerant circuit computing $f$. A crucial ingredient for securely soldering the garbled gates into circuits are XOR-homomorphic commitments which in [61] were realized using expensive Pedersen commitments [62]. In the follow-up construction of [35] these were replaced by an asymptotically more efficient construction, however the concrete communication overhead of the proposed commitment scheme was inadequate for the protocol to be competitive for realistic circuit sizes and parameters. In the recent works of [21], [34] this overhead has been improved to an optimal rate- 1 and the resulting UC-secure XOR-homomorphic commitment scheme is both asymptotically and concretely very efficient. Finally the work of [41] introduced a different primitive for LEGO soldering called XOR-Homomorphic Interactive Hash, which has some advantages over the commitment approach. However, the best instantiation of XOR-Homomorphic Interactive Hash still induces higher overall overhead than the commitment approach when using the schemes of [21], [34].

Although the original LEGO protocol, and the abovementioned follow-up works, asymptotically are very efficient, the overall consensus in the secure computation community has been that the reliance of XOR-homomorphic commitments for all circuit wires hinders actual practical efficiency. In this work we thoroughly investigate the practical efficiency of the LEGO approach and, in contrast to earlier beliefs, we demonstrate that it is indeed among the most practical protocols to date for general secure $2 \mathrm{PC}$ using garbled circuits.

\section{Our Contributions}

We implement the TinyLEGO protocol with added support for both independent and dependent preprocessing. Furthermore, our protocol supports fully reactive computation, meaning that when a function result has been obtained, another function depending on this result can be evaluated. Also, the independent preprocessing phase can be rerun at any

\begin{tabular}{lcccc}
\hline Setting & $\begin{array}{c}\text { Ind. } \\
\text { Preprocessing* }\end{array}$ & $\begin{array}{c}\text { Dep. } \\
\text { Preprocessing }\end{array}$ & $\begin{array}{c}\text { Online } \\
\text { Latency }\end{array}$ & $\begin{array}{c}\text { Online } \\
\text { Throughput }\end{array}$ \\
\hline $\begin{array}{l}\text { Single Execution } \\
\text { 1 x AES-128 }\end{array}$ & $89.61 \mathrm{~ms}$ & $13.23 \mathrm{~ms}$ & $1.46 \mathrm{~ms}$ & $1.46 \mathrm{~ms}$ \\
1 x SHA-256 & $478.54 \mathrm{~ms}$ & $164.40 \mathrm{~ms}$ & $11.19 \mathrm{~ms}$ & $11.19 \mathrm{~ms}$ \\
\hline Amortized & & & & \\
128 x AES-128 & $14.85 \mathrm{~ms}$ & $0.68 \mathrm{~ms}$ & $1.15 \mathrm{~ms}$ & $0.09 \mathrm{~ms}$ \\
128 x SHA-256 & $173.05 \mathrm{~ms}$ & $12.13 \mathrm{~ms}$ & $9.35 \mathrm{~ms}$ & $1.09 \mathrm{~ms}$ \\
\hline * Not including the time to compute the initial BaseOTs. & &
\end{tabular}

TABLE II. PERFORMANCE SUMMARY OF OUR PROTOCOL ON A HIGH BANDWIDTH (10GBIT) LAN NETWORK.

time if additional garbling material is necessary. As part of our prototype we also implement the XOR-homomorphic commitment scheme of [34] and report on its efficiency separately as we believe our findings can be of independent interest. This is to our knowledge the first implementation of a protocol based directly on the LEGO paradigm and of the mentioned commitment scheme. The support for independent preprocessing is achieved from the fact that the bulk of the computation using the LEGO approach is based on cut-andchoose of independently garbled gates and hence only depends on the security parameter and the number of AND gates one wishes to preprocess. The subsequent soldering phase can then be seen as a dependent preprocessing phase where knowledge of the circuit $f$ is required. This multi-level preprocessing is in contrast to previous non-LEGO protocols based on cut-andchoose of garbled circuits in the offline/online setting where the entire offline phase depends on the circuit to be evaluated. In more detail our main contributions are as follows:

1) We propose a new technique for dealing with the selective-OT attack on 2PC protocols based on garbled circuits. Our technique makes use of a globally correlated OT functionality $\left(\mathcal{F}_{\Delta-\text { ROT }}\right)$ combined with XORhomomorphic commitments and a Free-XOR garbling scheme [47]. Using the well-known fact of Beaver [8] that OTs can be precomputed, we can mitigate the selectiveOT attack by having the circuit constructor decommit to a single value per input bit of the evaluator in the online phase. This ensures that if the constructor tries to cheat, the evaluator aborts regardless of the value of his input. The technique is general and we believe that it can be used in other 2PC protocols based on garbled circuits as well. We also provide a more efficient instantiation of $\mathcal{F}_{\Delta \text {-ROT }}$ than previously appearing in the literature by tightening the analysis of the construction presented in [19].

2) As part of our 2PC prototype we also implement the XOR-homomorphic commitment scheme of [34]. It is already known that this scheme is asymptotically very efficient, but this is to our knowledge the first time its practical efficiency has been thoroughly investigated. The result is a very efficient scheme achieving an amortized cost of less than a microsecond for both committing and decommitting to a random value. To maximize performance we utilize cache efficient matrix-transposition and inspired by the construction of [21] we use the Intel Streaming SIMD Extension (SSE) instruction PCLMULQDQ to efficiently compute the required linear combinations.

3) We build our LEGO prototype on top of the abovementioned implementation which results in a very effi- 
cient and flexible protocol for maliciously secure reactive 2PC. As our online phase consists of an optimal two rounds, we can securely evaluate an AES-128 with latency down to $1.13 \mathrm{~ms}$. When considering throughput we can do each AES-128 block in amortized online time $0.08 \mathrm{~ms}$ (considering 1024 blocks). In applications where independent preprocessing can be utilized our offline phase is superior to all previous $2 \mathrm{PC}$ protocols, in particular based on our experiments we see a 6-54x gain over [65] depending on network and number of circuits considered. If preprocessing is not applicable, for most settings we cannot compete with the offline phase of [65], but note that the difference is within a factor 1.2-3x. See Table II for an overview of our performance in different settings and Section VI for a more detailed presentation and comparison of our results.

\section{PRELIMINARIES}

In this section we give some of the technical background for LEGO garbling, adopting the notation and conventions of the original TinyLEGO protocol [33] for ease of exposition.

\section{A. Notation}

We will use as shorthand $[n]=\{1,2, \ldots, n\}$ and $[i ; j]=$ $\{i, i+1, i+2, \ldots, j\}$. We write $e \in_{R} S$ to mean: sample an element $e$ uniformly at random from the finite set $S$. We sometimes (when the semantic meaning is clear) use subscript to denote an index of a vector, i.e., $x_{i}$ denotes the $i$ 'th bit of a vector $x$. We use $k$ to denote the computational security parameter and $s$ to represent the statistical security parameter. Technically, this means that for any fixed $s$ and any polynomial time bounded adversary, the advantage of the adversary is $2^{-s}+\operatorname{negl}(k)$ for a negligible function negl. i.e., the advantage of any adversary goes to $2^{-s}$ faster than any inverse polynomial in the computational security parameter. If $s=\Omega(k)$ then the advantage is negligible. In our experiments we set $k=128$ and $s=40$.

\section{B. Circuit Conventions}

We assume $A$ is the party constructing the garbled gates and call it the constructor. Likewise, we assume B is the party evaluating the garbled gates and call it the evaluator. Furthermore, we say that the functionality they wish to compute is $z=f(x, y)$, where $\mathrm{A}$ gives input $x$ and $\mathrm{B}$ gives input $y$. We assume that $f$ is described using only NOT, XOR and AND gates. The XOR gates are allowed to have unlimited fan-in, while the AND gates are restricted to fan-in 2, and NOT gates have fan-in 1. All gates are allowed to have unlimited fan-out. We denote the bit-length of $x$ by $|x|=n_{\mathrm{A}}$, the bit-length of $y$ by $|y|=n_{\mathrm{B}}$ and let $n=n_{\mathrm{A}}+n_{\mathrm{B}}$. We will denote the bitlength of the output $z$ by $|z|=m$. Furthermore, we assume that the first $n_{\mathrm{A}}$ input wires are for A's input and the following $n_{\mathrm{B}}$ input wires are for B's input.

We define the semantic value of a wire-key of a garbled gate to be the bit it represents. We will use $K_{j}^{b}$ to denote the $j$ 'th wire key representing bit $b$. Sometimes, when the context allows it, we will let $L_{g}^{b_{l}}, R_{g}^{b_{r}}$, and $O_{g}^{b_{o}}$ denote the left input, right input, and output key respectively for garbled gate $g$ representing the bits $b_{l}, b_{r}$ and $b_{o}$. When the bit represented by a key is unknown we simply omit the superscript, e.g. $K_{j}$.

\section{Free-XOR and Soldering}

The LEGO protocols [35], [61] and [33] all assume that the underlying garbling scheme supports the notion of FreeXOR [47], meaning that the XOR of the 0- and 1-key on any wire of any garbled gate yields the same value, $\Delta$, which we call the global difference. In addition to making garbling and evaluating XOR gates virtually free, this optimization also allows for easily soldering wires together. A soldering of two wires is a way of transforming a key representing bit $b$ on one wire into a key representing bit $b$ on the other wire. As we will see in more detail below, with Free-XOR, a soldering is simply the XOR of the 0-keys on the two wires. Furthermore, in order to avoid any cheating all wires of all garbled gates are committed to using a XOR-homomorphic commitment functionality $\mathcal{F}_{\mathrm{HCOM}}$ and the solderings are then always decomitted when needed.

As an example, assume we wish to solder the output wire of gate $g$ onto the left input wire of gate $g+1$. In doing so we decommit the value $S_{g+1}^{L}=O_{g}^{0} \oplus L_{g+1}^{0}$ using $\mathcal{F}_{\text {HCOM }}$. When gate $g$ outputs the key representing the bit $b$ one can now learn the left $b$-key for gate $g+1$. Specifically it can be computed as $O_{g}^{b} \oplus S_{g+1}^{L}=\left(O_{g}^{0} \oplus(b \cdot \Delta)\right) \oplus O_{g}^{0} \oplus L_{g+1}^{0}=$ $L_{g+1}^{0} \oplus(b \cdot \Delta)=L_{g+1}^{b}$. This obviously generalizes when one wishes to solder together several wires, e.g. if we wish to solder the output wire of gate $g$ to the left input wire of gate $g+1$, $g+2$ and $g+3$, then it is enough to decommit the values $S_{g+1}^{L}=O_{g}^{0} \oplus L_{g+1}^{0}, S_{g+2}^{L}=O_{g}^{0} \oplus L_{g+2}^{0}, S_{g+3}^{L}=O_{g}^{0} \oplus L_{g+3}^{0}$.

It is also straightforward to evaluate XOR gates as part of the soldering: To compute the XOR of $g$ and $g+1$ and then use this result as the left input to gate $g+2$ we decommit the value $S_{g+2}^{L}=\left(O_{g}^{0} \oplus O_{g+1}^{0}\right) \oplus L_{g+2}^{0}$. We see now that $O_{g}^{a} \oplus O_{g+1}^{b} \oplus S_{g+2}^{L}=a \cdot \Delta \oplus b \cdot \Delta \oplus L_{g+2}^{0}=L_{g+2}^{a \oplus b}$ as desired. In conclusion a soldering is therefore always the XOR of the 0-keys of the wires going into an XOR gate and the 0-key of the wire we wish to solder the result onto.

\section{A NeW ApproACH to Eliminate the SELECTIVE-OT ATTACK}

As already mentioned in Section I the selective-OT attack enables a corrupt $A$ to learn any input bit of $B$ with success probability $1 / 2$ for each bit. Prior work has dealt with this attack in different ways, but the off-the-shelf blackbox solution has typically been the $s$-probe resistant matrix approach of [51], [56], [67]. These approaches augment the evaluation circuit $f \rightarrow f^{\prime}$ so that learning any $s-1$ input bits of $\mathrm{B}$ in $f^{\prime}$ leaks nothing about the actual input used in the original $f$. The downside of this approach is that the input length of $\mathrm{B}$ needs to be increased to $n_{\mathrm{B}}^{\prime}>n_{\mathrm{B}}$, which in turn results in more communication, computation and OTs. For the approach of [51] and [56] the increase is to $n_{\mathrm{B}}^{\prime}=n_{\mathrm{B}}+\max \left(4 n_{\mathrm{B}}, 20 s / 3\right)$ while for the approach of [67] we have $n_{\mathrm{B}}^{\prime} \leq n_{\mathrm{B}}+\lg \left(n_{\mathrm{B}}\right)+n_{\mathrm{B}}+s+s \cdot \max \left(\lg \left(4 n_{\mathrm{B}}\right), \lg (4 s)\right)$. In addition to extending the input size, experiments of [56] show that producing the $s$-probe resistant version of $f$ can be a computationally expensive task (up to several seconds for 1000-bit input). 


\section{A. Our New Approach}

We propose a new approach that combines the use of 1out-of-2 $\Delta$-ROTs (also called globally correlated OTs), XORhomomorphic commitments and the Free-XOR technique that sidesteps the need of expanding the input size of $B$ as described above. We recall that $\Delta$-ROTs are similar to Random OTs (ROT), except that all OTs produced are correlated with a global difference $\Delta$. In other words, for each $\Delta$-ROT $i$ produced, the following relation holds for a fixed $\Delta: r_{i}^{1}=r_{i}^{0} \oplus \Delta$ where $r_{i}^{0}, \Delta \in\{0,1\}^{k}$ are uniformly random strings known to the sender and $b_{i} \in\{0,1\}$ is the uniformly random choice-bit of the receiver who learns $r_{i}^{b_{i}}$ as part of the OT protocol. Our approach is described below and is inspired by the protocol of Beaver [8] for precomputing OT.

1) The parties precompute $\left(n_{\mathrm{B}}+s\right) \Delta$-ROTs such that the sender learns $\left(\Delta, r_{i}^{0}\right)$ and the receiver learns $\left(r_{i}^{b_{i}}, b_{i}\right)$ for $i \in\left[n_{\mathrm{B}}+s\right]$. The sender will now commit, using the XORhomomorphic commitment scheme, to $\Delta$ and each $r_{i}^{0}$. In order to verify that the sender indeed committed to the $\Delta$ used in the OTs, the parties run a simple check in the following way: B sends $\left\{\left(r_{j}^{b_{j}}, b_{j}\right)\right\}_{j \in\left[n_{\mathrm{B}} ; n_{\mathrm{B}}+s\right]}$ to A which in turn needs to successfully decommit to the received values. The $s$ OTs used for the check are hereafter discarded. The reason why $B$ needs to send the values to $A$ in the first place is that it needs to prove knowledge of the value $r_{j}^{b_{j}}$ before it is safe for $\mathrm{A}$ to open it. For each of the $s$ tests, if A did not commit to the $\Delta$ used in the OTs, then it can only pass the test with probability at most $1 / 2$ as the choice-bits of $\mathrm{B}$ are uniformly random. Because these are also chosen independently we see that the check therefore catches a cheating $A$ with overwhelming probability $1-2^{-s}$.

2) If the check succeeds, $A$ uses the $\Delta$ learned from the OTs above as the global difference in the Free-XOR garbling scheme. Recall that this means that all garbling keys will be correlated in the same way as the $\Delta$-ROTs, i.e. $K_{l}^{1}=$ $K_{l}^{0} \oplus \Delta$ for all $l$. In particular this is the case for the keys associated to the input of $\mathrm{B}$ which need to be obliviously transferred in the online phase. In addition, in our 2PC protocol all 0-keys $K_{l}^{0}$ have been committed to using the same XOR-homomorphic commitment scheme as used for the OT strings $r_{i}^{0}$ and $\Delta$.

3) Finally when $B$ learns its real input $y$, it computes $e=y \oplus b$ and sends this to $\mathrm{A}$ where $b$ are the choicebits used in the precomputed $\Delta$-ROTs. A will respond by decommitting the values $\left\{D_{i}=K_{i}^{0} \oplus r_{i}^{e_{i}}\right\}_{i \in\left[n_{\mathrm{B}}\right]}$. B can now compute its actual input keys $K_{i}^{y_{i}}=D_{i} \oplus r_{i}^{b_{i}}=$ $K_{i}^{0} \oplus r_{i}^{e_{i}} \oplus r_{i}^{b_{i}}=K_{i}^{0} \oplus y_{i} \cdot \Delta$.

The above approach eliminates the selective-OT attack as the only way a corrupt $\mathrm{A}$ can cheat is by committing to different values ${r^{\prime}}_{i}^{0} \neq r_{i}^{0}$ where $r_{i}^{0}$ is the value sent using the $\Delta$-ROTs. However if this is the case then $D_{i}^{\prime} \oplus r_{i}^{b_{i}} \notin\left\{K_{i}^{0}, K_{i}^{1}\right\}$ and B will abort regardless of the value of his input $y_{i}$. One caveat of the above approach is that it allows a corrupt $A$ to flip an input bit $i$ of B without getting caught by committing to $r_{i}^{0} \oplus \Delta$ instead of $r_{i}^{0}$. In our 2PC protocol we eliminate this issue by ensuring that $\operatorname{lsb}(\Delta)=1$ and by securely leaking $\operatorname{lsb}\left(r_{i}^{0}\right)$ to $\mathrm{B}$. This allows B to check that the resulting key $K_{i}=D_{i} \oplus r_{i}^{b_{i}}$ indeed carries the correct value $y_{i}$, by verifying that

$$
\begin{aligned}
y_{i} & =\operatorname{lsb}\left(K_{i}\right) \oplus \operatorname{lsb}\left(D_{i}\right) \oplus \operatorname{lsb}\left(r_{i}^{0}\right) \oplus e_{i} \\
& =\operatorname{lsb}\left(K_{i}\right) \oplus \operatorname{lsb}\left(K_{i}^{0}\right) \oplus \operatorname{lsb}\left(r_{i}^{e_{i}}\right) \oplus \operatorname{lsb}\left(r_{i}^{0}\right) \oplus e_{i} \\
& =\operatorname{lsb}\left(K_{i}\right) \oplus \operatorname{lsb}\left(K_{i}^{0}\right) .
\end{aligned}
$$

This secure leaking is described in the VerLeak step of Fig. 1 and the check is carried out as part of the Eval step of Fig. 2, both of which are presented in Section IV.

\section{B. On Constructing $\Delta$-ROTs}

The above technique requires $\left(n_{\mathrm{B}}+s\right) \Delta$-ROTs to obliviously transfer the input keys of B. However, current stateof-the-art protocols for OT extension [6], [19], [43], [60] all produce ROTs. It can be seen by inspecting the above OT extension protocols that they all produce a weaker variant of $\Delta$-ROT called leaky $\Delta$-ROT as an intermediate step. The leaky $\Delta$-ROT is identical to $\Delta$-ROT in that all OT pairs are correlated with a global $\Delta$, however a corrupt receiver can cheat and learn some bits of $\Delta$ with non-negligible probability. In fact, for each bit learned of $\Delta$, the receiver gets caught with probability $1 / 2$, which means it can learn up to $s-1$ bits of $\Delta$, while the other bits remain uniformly random in its view. The work of [19] gives a construction for $\Delta$-ROTs of string length $v$ from leaky $\Delta$-ROTs of string length $22 v / 3 \sim 7.33 v$ using linear randomness extraction. Concretely, they propose multiplying all the strings learned from the leaky $\Delta$-ROT protocol with a random matrix $A \in\{0,1\}^{22 v / 3 \times v}$. In this work we observe that the factor $22 / 3$ is not tight and by applying Theorem 1 below we can reduce the number of rows in $A$ down to $v+s$, going from a multiplicative to an additive factor.

Theorem 1 ([73], Theorem 7). Let $X=x_{1}, x_{2}, \ldots, x_{u}$ be a binary sequence generated from a bit fixing source in which $l$ bits are unbiased and independent, the other $u-l$ bits are fixed or copies of the $l$ independent random bits. Let $A$ be a $u \times v$ random matrix such that each entry of $A$ is 0 or 1 with probability $1 / 2$. Given $Y=X A$, then we have that

$$
\operatorname{Pr}_{A}[\rho(Y) \neq 0] \leq 2^{v-l}
$$

where $\rho(Y)$ is defined as the statistical distance to the uniform distribution over $\{0,1\}^{v}$, i.e. $\rho(Y)=\frac{1}{2} \sum_{y \in\{0,1\}^{v}} \mid \operatorname{Pr}[Y=$ $y]-2^{-v}$.

Now let $u=v+s$ and let $\Delta$ have length $u$ and let $\Delta A$ have length $v$. Consider an adversary $B$ who is allowed to try to learn some of the bits of $\Delta$ to make $\Delta A$ non-uniform. In our setting, if an adversary $B$ tries to learn $\lambda$ bits of $\Delta$ it is caught except with probability $2^{-\lambda}$. If $B$ is not caught then it learns $\lambda$ bit positions and the remaining bits are independent and uniform. Since we have $u=v+s$, the $l$ in the above theorem equals $u-\lambda=v+(s-\lambda)$ when $B$ learns $\lambda$ bits, i.e., $2^{v-l}=2^{\lambda-s}$. This implies that for all $0 \leq \lambda<s$ it holds that the probability that $B$ is not caught and at the same time $\Delta A$ is not uniform is at most $2^{-\lambda} 2^{\lambda-s}=2^{-s}$. Clearly, for all $\lambda \geq s$, then the probability that $B$ is not caught and at the same time $\Delta A$ is not uniform is at most the probability $B$ is not caught, which is at most $2^{-s}$. This shows that when $u=v+s$ then for all $B$, the probability that $B$ is not caught and at the same time $\Delta A$ is not uniform is at most $2^{-s}$, which is negligible. 
The consequence of our new analysis is that we can choose the random matrix as $A \in\{0,1\}^{(v+s) \times v}$ and thus we only have to produce leaky $\Delta$-ROTs of length $v+s$ instead of length $22 v / 3$, a substantial optimization. As we ultimately require (non-leaky) $\Delta$-ROTs of length $k$, we can utilize any of the mentioned OT extension protocols to produce leaky $\Delta$-ROTs of length $k+s$ and then apply the linear randomness extraction on the resulting OT-strings. For the parameters $s=40$ and $k=128$ considered in this work our refined analysis ultimately yields an improvement of around a factor 5.6x compared to the previous best known result of [19].

\section{The PROTOCOL}

As already mentioned in Section I, our protocol is based on TinyLEGO [33], but modified to support preprocessing of all garbled components along with our new approach for dealing with the selective-OT attack of Section III-A. This includes removing the restriction of $\mathrm{B}$ choosing input and committing to the cut-and-choose challenges before obtaining the garbling material and solderings. As a consequence, our modifications allow for multi-leveled preprocessing and a very efficient online phase. We give a description of our resulting protocol in Fig. 1 and Fig. 2. See [33] for a more detailed specification of the original TinyLEGO protocol. At a high level our protocol can be broken down into four main steps.

1) The Setup phase initializes the commitment scheme. All public-key operations of our protocol can be carried out in this initial step, including the BaseOTs required for bootstrapping OT extension.

2) The Generate step takes as input the number of gates $q$, number of inputs $n$ and number of outputs $m$ the parties wish to preprocess. After sending the garbled gates and wire authenticators and committing to all associated wires, a cut-and-choose step is run between the parties. The wire authenticators is a gadget that either accepts or rejects a given key (without revealing the value of the key) and it was shown in [33] that constructing AND buckets from both garbled gates and wire authenticators can significantly reduce the overall communication compared to using garbled gates alone. After the cut-and-choose step, using the XOR-homomorphic commitments, the parties solder the remaining garbled gates and wire authenticators randomly into independent fault tolerant AND buckets.

3) The Build step takes as input the circuit description $f$ and through the XOR-homomorphic commitments, A sends the required solderings to glue together a subset of previously produced AND buckets so that they compute $f$.

4) Finally, the Eval step depends on the parties' inputs to $f$. It consists of two rounds, first B sends a correction value $e$ which depends on his input $y$, and as a response $A$ decommits to B's masked input keys as well as sending it's own input keys directly. Finally $A$ also decommitments to the lsb of all output 0-keys. This allows B to evaluate the garbled circuit and decode the final output.

We highlight that our modified protocol also naturally supports the notion of streaming or pipelining of garbled circuit evaluation [39] which was not the case in [33]. This can be seen by the fact that one can evaluate the circuit $f$ in a layered approach and using the XOR-homomorphic commitments to glue the output of one layer onto the input of the next layer.
The Setup step is only required to be run once, regardless of the number of future calls to Generate.

$\operatorname{Setup}(\mathbf{p p})$ :

1) On input $\left(k, s, p_{g}, p_{a}, \beta, \alpha, \tilde{\beta}, \tilde{\alpha}\right) \leftarrow \mathrm{pp}, \mathrm{A}$ and $\mathrm{B}$ initialize the functionality $\mathcal{F}_{\mathrm{HCOM}}$ by sending (init, sid, $\left.\mathrm{A}, \mathrm{B}, \kappa\right)$ to it, where $\kappa$ is the key-length of the garbling scheme.

The Generate step produces $q$ garbled AND gates which can be soldered into circuits that in total can have $n$ inputs and $m$ outputs. It is possible to do multiple calls to Generate at any point in time in order to produce more garbling material.

$\operatorname{Generate}(q, n, m)$ :

1) Let $Q$ and $A$ be chosen such that after running the below cutand-choose step, with overwhelming probability $(q \beta+n \tilde{\beta})$ garbled gates and $(q \alpha+n \tilde{\alpha})$ wire authenticators survive.

2) $\mathrm{A}$ and $\mathrm{B}$ invoke $\mathcal{F}_{\Delta \text {-ROT }}(n+s)$ times from which $\mathrm{A}$ learns $\Delta$ and random strings $r_{i}^{0}$ and $\mathrm{B}$ learns the choice-bits $b_{i}$ and $r_{i}^{b_{i}}$ for $i \in[n+s]$. Furthermore, $\mathrm{A}$ instructs $\mathcal{F}_{\Delta \text {-ROT }}$ to ensure that $\operatorname{lsb}(\Delta)=1$.

3) Next, A garbles $Q$ AND gates and constructs $A$ wire authenticators using $\Delta$ and sends these to B.

4) A then commits to each wire of the garbled AND gates, each authenticated wire produced, $\Delta$, the 0 -strings received from $\mathcal{F}_{\Delta \text {-ROT }}$, and $m+s$ random values $\left\{v_{j}\right\}_{[m+s]}$. Thus it sends $3 Q+A+1+n+s+m+s$ values to $\mathcal{F}_{\mathrm{HCOM}}$.

VerLeak:

5) For $i \in[n]$ and $j \in[m+s], \mathrm{A}$ sends $\operatorname{lsb}\left(r_{i}^{0}\right)$ and $\operatorname{lsb}\left(v_{j}\right)$ to $B$.

6) $\mathrm{B}$ challenges $\mathrm{A}$ to send, using $\mathcal{F}_{\mathrm{HCOM}}, s$ random linear combinations of $r_{i}^{0}, v_{j}$ and $\Delta$ for $i \in[n]$ and $j \in[m]$. Also, the $l$ 'th combination is set to include a one-time blinding value $v_{m+l}$ for $l \in[s]$.

7) B verifies that $l s b$ of the received $s$ values correspond to the same linear combinations of the initial lsb values sent by $A$ in step 5. In addition, if $\Delta$ is included in a linear combination $B$ flips the value. This ensures that indeed $\operatorname{lsb}(\Delta)=1$.

Cut-and-Choose:

8) After receiving the garbled gates (wire authenticators), B chooses to check any gate (wire authenticator) with probability $p_{g}\left(p_{a}\right)$. B then challenges $\mathrm{A}$ to send, using $\mathcal{F}_{\mathrm{HCOM}}$, two random inputs and the corresponding AND output of the selected garbled gates and a random input of the selected wire authenticators.

9) B evaluates the selected garbled gates and wire authenticators and checks that they output the received output key and that they verify the values received from $\mathcal{F}_{\text {HCOM }}$, respectively.

10) In addition, for $i \in[n ; n+s] \mathrm{B}$ sends $r_{i}^{b_{i}}=r_{i}^{0} \oplus\left(b_{i} \cdot \Delta\right)$ to $A$ which in turn instructs $\mathcal{F}_{\text {HCOM }}$ to send back the same value. This is to ensure that the committed $\Delta$ is the one used in $\mathcal{F}_{\Delta \text {-ROT. }}$.

Bucketing:

11) For the remaining garbled gates (wire authenticators), B samples and sends a random permutation that fully describes how these are to be combined into $q$ AND buckets of size $\beta+\alpha, n$ input buckets of size $\tilde{\beta}$ and $n$ input authenticators of size $\tilde{\alpha}$.

12) A then sends, using $\mathcal{F}_{\mathrm{HCOM}}$, all the required solderings such that for all the specified bucket gadgets, each component is defined with the same input/output keys.

Fig. 1. The modified TinyLEGO protocol with support for preprocessing in the $\left(\mathcal{F}_{\text {HCOM }}, \mathcal{F}_{\Delta \text {-ROT }}\right)$-hybrid model (Part 1). 
The Build step uses the garbling material created in Generate to construct a fault tolerant garbled circuit computing $f$.

$\operatorname{Build}(f)$ :

1) $\mathrm{A}$ instructs $\mathcal{F}_{\mathrm{HCOM}}$ to send the required solderings such that the first $|f|$ unused AND buckets correctly compute $f$. This includes the solderings to attach $n_{\mathrm{A}}$ input buckets and $n$ input authenticators onto the final garbled circuit.

In the Eval step the parties transfer to B all input keys in an oblivious manner which then allows $B$ to evaluate and decode the garbled circuit previously constructed using the Build step.

$\operatorname{Eval}(x, y)$ :

1) For input $y \in\{0,1\}^{n_{\mathrm{B}}}$, B sends $e=b \oplus y$ to A, where $b$ is the first $n_{\mathrm{B}}$ unused choice-bits of $\mathcal{F}_{\Delta \text {-ROT }}$.

2) A then instructs $\mathcal{F}_{\mathrm{HCOM}}$ to send to the values $\left\{D_{i}=r_{i}^{0} \oplus\right.$ $\left.K_{i}^{0} \oplus e_{i} \cdot \Delta\right\}_{i \in\left[n_{\mathrm{B}}\right]}$ where $K_{i}^{0}$ is the 0 -key on the $i$ 'th input wire of $\mathrm{B}$ and $r_{i}^{0}$ is the first unused $\Delta$-ROT string. Also, for the input $x \in\{0,1\}^{n_{\mathrm{A}}}$, it sends the corresponding input keys $\left\{K_{i}^{x_{i}}\right\}_{i \in\left[n_{\mathrm{A}}\right]}$ directly to B.

3) Finally, $A$ instructs $\mathcal{F}_{\text {HCOM }}$ to send the output decoding values $\left\{D_{j}=v_{j}^{0} \oplus K_{j}^{0}\right\}_{j \in[m]}$ to $\mathrm{B}$ where $K_{j}^{0}$ is the $j$ 'th output 0-key of the garbled circuit and $\left\{v_{j}\right\}_{j \in[m]}$ are the first $m$ unused blinding values setup in the VerLeak step.

4) Upon receiving the above, for $i \in\left[n_{\mathrm{B}}\right] \mathrm{B}$ computes $K_{n_{\mathrm{A}}+i}=$ $r_{i}^{b_{i}} \oplus D_{i}$ and $\operatorname{lsb}\left(K_{i}^{0}\right)=\operatorname{lsb}\left(D_{i}\right) \oplus \operatorname{lsb}\left(r_{i}^{0}\right) \oplus e_{i}$ and verifies that $\operatorname{lsb}\left(K_{n_{\mathrm{A}}+i}\right) \oplus \operatorname{lsb}\left(K_{i}^{0}\right)=y_{i}$. Then using the input authenticators, B also verifies that the keys $\left\{K_{i}\right\}_{i \in\left[n_{\mathrm{A}}\right]}$ of $\mathrm{A}$ are valid input keys to the garbled circuit.

5) If everything checks out, $B$ evaluates the previously constructed garbled circuit on the input keys $\left(K_{1}, K_{2}, \ldots, K_{n}\right)$ to obtain the output keys $\left(Z_{1}, Z_{2}, \ldots, Z_{m}\right)$. For $j \in[m]$ it then computes $d_{j}=\operatorname{lsb}\left(v_{j}\right) \oplus \operatorname{lsb}\left(D_{j}\right)$ and decodes $z_{j}=\operatorname{lsb}\left(Z_{j}\right) \oplus d_{j}$. Finally, B outputs $z=\left(z_{1}, z_{2}, \ldots, z_{m}\right)$.

Fig. 2. The modified TinyLEGO protocol with support for preprocessing in the $\left(\mathcal{F}_{\text {HCOM }}, \mathcal{F}_{\Delta \text {-ROT }}\right)$-hybrid model (Part 2$)$.

Each of these layers can be processed on the fly with our protocol and in this way the circuit never needs to be stored entirely at any given time. This approach is similar to that proposed in [59] for reusing garbled values, however in this setting everything works out-of-the-box due to the XORhomormophic commitments on all circuit wires.

The LEGO approach also has the advantage compared to traditional cut-and-choose protocols that only a single fault tolerant garbled circuit is produced and evaluated. This removes the necessity of ensuring input consistency for all the evaluation circuits. It also sidesteps the overhead of transferring multiple sets of input keys in the online phase, one set for each evaluation circuit.

\section{A. Bucketing}

With the bucketing approach of [33] each AND bucket consists of $\beta$ garbled gates and $\alpha$ wire authenticators. For any garbled gate (wire authenticator) the probability $p_{g}\left(p_{a}\right)$ is used to determine if it is checked in the cut-and-choose or not. The value of $p_{g}$ and $p_{a}$ therefore induces a certain sense of "quality" level of the remaining non-checked garbled components which affects the required bucketing size. In addition there are also the special cases of input buckets and input authenticators, which are buckets that consist of garbled gates only $(\operatorname{size} \tilde{\beta})$ and wire authenticators only (size $\tilde{\alpha}$ ), respectively. These are attached to the input wires of the final garbled circuit and serve to guarantee validity of the input keys, along with guaranteeing that $B$ can always learn the final output $f(x, y)$, even if $\mathrm{A}$ is cheating. This is so since the input buckets can be seen as a trapdoor that together with the global difference $\Delta$ allows B to extract the input $x$ of $\mathrm{A}$. It is then clear that it can compute $f(x, y)$ directly. These special buckets are necessary as our regular AND buckets do not rule out outputting both the 0 and 1-key (say if one of the garbled gates in the bucket is in fact a NAND gate). However, if a bucket outputs two distinct keys it is guaranteed that they are both valid and hence their XOR is $\Delta$ and B can extract $x$. If no cheating is detected then the input buckets are simply ignored by $\mathrm{B}$.

As already established in the original LEGO paper [61], the number of AND gates $q$ directly affects the required size of the buckets, meaning that as $q$ grows the required bucket size can be decreased while still retaining the same level of security. Theorem 2 below gives a direct way of computing the success probability of a corrupt $A$ given the parameters $q, n, \beta, \alpha, \tilde{\beta}, \tilde{\alpha}$.

Theorem 2 ([33], Lemma 9). Given the bucketing parameters $q, n, \beta, \alpha, \tilde{\beta}, \tilde{\alpha}$ for the case where $\alpha=\beta-1$ we can bound the probability of the bad bucketing events occurring as:

$$
\begin{gathered}
q \cdot\left(\prod_{i=\beta}^{1} \operatorname{Pr}\left[\frac{\left(1-p_{g}\right) 4 i}{p_{g}(q \beta+n \tilde{\beta})+\left(1-p_{g}\right) 4 i}\right)+\right. \\
\sum_{l=2}^{\beta} \prod_{i=\beta}^{l}\left(\frac{\left(1-p_{g}\right) 4 i}{p_{g}(q \beta+n \tilde{\beta})+\left(1-p_{g}\right) 4 i}\right) \cdot \\
\left.\prod_{j=\alpha}^{\alpha+2-l}\left(\frac{\left(1-p_{a}\right) 2 j}{p_{a}(q \alpha+n \tilde{\alpha})+\left(1-p_{a}\right) 2 j}\right)\right) \\
\operatorname{Pr}[\text { Any bad input authenticator }] \leq \\
\left\lceil\cdot \sum_{v=1}^{\left\lceil\frac{\tilde{\alpha}}{2}\right\rceil} \prod_{l=\tilde{\alpha}}^{v}\left(\frac{\left(1-p_{a}\right) 2 l}{p_{a}(q \alpha+n \tilde{\alpha})+\left(1-p_{a}\right) 2 l}\right)\right. \\
\operatorname{Pr}[\text { Any bad input bucket }] \leq \\
\left\lceil\cdot \sum_{l=1}^{\left\lceil\frac{\tilde{\beta}}{2}\right\rceil} \prod_{i=\tilde{\beta}}^{l}\left(\frac{\left(1-p_{g}\right) 4 i}{p_{g}(q \beta+n \tilde{\beta})+\left(1-p_{g}\right) 4 i}\right)\right.
\end{gathered}
$$

Based on Theorem 2, given the number of AND gates $q$ and the number of inputs $n$ we directly compute the optimal choices of $\beta, \alpha, \tilde{\beta}, \tilde{\alpha}$ for minimizing the overall communication of the protocol while still guaranteeing a negligible upper bound on the success probability of a corrupt $A$. This is a once and for all computation so for our implementation we have precomputed a table of secure choices using a simple script which is looked up on runtime when $q$ and $n$ have been decided. We note that it is also possible to minimize for lowest possible bucket size if desired. This has the effect of reducing the computational overhead in the online phase at the price of 
increasing both communication and computational overhead in the independent preprocessing phase. In our experiments in Section VI we solely minimize for overall communication.

\section{B. Security}

Our protocol is similar to the TinyLEGO protocol in [33] and the proof follows the same general outline. We will therefore only give a very brief sketch of the overall proof strategy and then describe how to deal with the changes we made relative to TinyLEGO.

Consider first the case where the garbler $\mathrm{A}$ is corrupted. As is typically the case it is easy to see that the communication of the protocol does not leak any information on the input of $\mathrm{B}$ as long as the protocol does not abort. The garbler $\mathrm{A}$ might however give wrong input to some of the OTs used by $B$ to choose its input keys, giving rise to selective errors where the abort probability depends on the input of $B$. The garbler $A$ might also create some bad garbled gates which could a priori result in an abort or a wrong output, which might both leak information on the input of $\mathrm{B}$. The problem with bad gates is handled exactly as in [33], by setting the cut-and-choose parameters and bucket sizes appropriately. We however handle the case with bad inputs to the OTs differently, as described below. In the universal composability (UC) framework [20], when $A$ is corrupt, we also need to be able to extract the input of the corrupted $A$ from its communication and input to ideal functionalities (OT and commitment). We handle this exactly as [33]: the cut-and-choose ensures that most key authenticators only accept their two corresponding committed keys. For a good key authenticator the accepted key can then be compared to the committed values to compute its semantic value. The bucket size has been set such that there is a majority of good key authenticators on all input wires. This allows to compute the semantic of any accepted key by taking majority.

Consider then the case where the evaluator B is corrupted. As is typically the case, the communication clearly does not leak information to $B$ about the input of $A$. All that is left is therefore to describe how to handle two technical requirements imposed by the UC framework. First, we have to describe how to extract the input $y$ of a corrupted B. Second, after learning $y$ and $z=f(x, y)$ we must enforce that the simulated protocol constructs a circuit that evaluates to $z$. This must be done without knowing $x$. Extracting $y$ is handled exactly as in [33]. We simply inspect which choice bits B uses in the OTs for selecting its input. Hitting $z$ in the simulation is also handled exactly as in [33]. We simply construct the circuit correctly and run with input 0 for $A$. This gives a potentially wrong output $z^{\prime}=f(0, y)$. We patch this by giving appropriately chosen wrong output decoding information by opening a wrong least significant bit of the output key for the output wires $i$ where $z_{i}^{\prime} \neq z_{i}$. This is possible as the simulator controls the ideal functionality for commitment in the simulation.

We now focus on the changes we made to [33].

Change 1 In both protocols the output decoding information consists of the least significant bit of the output keys, securely leaked via the commitment scheme. However, the implementation differs. We use a noninteractive implementation which is slightly heavier on communication. In [33] they use an interactive protocol with less communication.

Change 2 In [33] they let B commit to the cut-and-choose challenges and choose his input via OT before obtaining the garbled gates, wire authenticators and solderings. We have removed this step. Now B picks his input after the circuit is constructed and does not commit to his challenges.

Change 3 In our protocol we take the global $\Delta$-value output by the OT extension and reuse it as the global $\Delta$ in the Free-XOR garbling scheme. In [33] they use two independent values.

Change 4 We protect against selective error on the input of B by using the same $\Delta$ in OT extension and garbling and using a $\Delta$-ROT to offer the input keys to $B$. We also leak the least significant bit of the $\Delta$-OT 0 strings to $B$ for all his input wires. In [33] a different technique was used.

Change 1 does not affect security. It was introduced to give better execution time for typical circuits.

We now address Change 2. The reason why B commits to the cut-and-choose challenges and chooses his input via OT before obtaining the garbled material in [33] is that security is proven via a reduction to a standard (non-adaptive) selective garbling scheme (e.g. [12]), where the adversary in the security game must supply its input before it gets the garbled circuit. Therefore they need to be able to extract the input and cut-andchoose challenges of $\mathrm{B}$ before assembling the garbled circuit in the simulation. We have skipped this step as it would prevent independent preprocessing. Now that we assemble the circuit before $B$ picks its input, the hope would be that we could do a reduction to an adaptive garbling scheme. However, due to the soldering approach of LEGO where the XOR of 0keys are sent to the evaluator before the input is determined, it is unclear how to reduce security to the standard notion of adaptive garbling, as some of these 0-keys are not known to the simulator. To overcome this we instead identify the defining property we need from the underlying garbling function which is that the outputs of the hash function appear random as long as the inputs are all unknown and have sufficient entropy, even if the inputs are related. A non-extractable, non-programmable random oracle clearly satisfies this property [13]. The type of garbling scheme considered in this work [72] could in principle be made adaptively secure by using a programmable random oracle using techniques described in [11], [55]. ${ }^{1}$ We avoid using the programability of the random oracle by changing the usual approach a little. Normally security proofs need to program the circuit to hit the right output. We instead garble the circuits correctly and then program or equivocate the output decoding information to decode to the value we need to hit. Specifically we use equivocation of the UC commitment scheme to incorrectly open the least significant bit of the output keys when we need to hit a different value. Returning to the simulation, we therefore garble all gates and answer all cutand-choose challenges honestly. As we now know all garbling keys we can also open consistently to differences between 0keys for the remaining evaluation gates. Finally, in order to

\footnotetext{
${ }^{1}$ It is also possible to build an adaptively secure garbling scheme (with short input keys) using a non-programmable random oracle [9], but this particular scheme is not as efficient as the one of [72].
} 
make the complete soldered garbled circuit "hit" the output $z$ we equivocate the openings of the least significant bits of the output keys such that this becomes the decoded value. This is possible as the decoding information is only opened after we extract the input of the corrupt receiver. If the garbling is done using a random oracle this will have the same distribution as in the protocol.

For Change 3 we again use that we are in the random oracle model. The first step in the proof will be to go from the case where $\Delta$ is reused in the garbling scheme to the case where an independent $\Delta^{\prime}$ is used for garbling as in [33]. In this hybrid we also let $\mathrm{A}$ commit to $\Delta^{\prime}$. We then use equivocation of the commitment scheme to make the cut-and-choose proof that $\Delta^{\prime}=\Delta$ go through. Since $B$ only sees one key for each wire and has high entropy on $\Delta$ and $\Delta^{\prime}$, this change will be indistinguishable to $B$ if the output of the hash function appears random as long as the inputs are all unknown and have sufficient entropy, even if inputs are related. As above, a nonextractable, non-programmable random oracle clearly satisfies this property.

We finally address Change 4 . Using a $\Delta$-ROT to offer the input keys to $B$ ensures that when $A$ inputs the keys to the OT, either both are correct or both are incorrect. If both are incorrect, it will be detected by a key authenticator independently of the input of $B$. This means that the only remaining attack vector is for $A$ to swap the two correct keys. This is detected by B as B knows the least significant bit of the $\Delta$-ROT 0-strings and the two correct keys have different least significant bits. Again the detection is independent of the input of $\mathrm{B}$. Notice that the output decoding information is sent to $\mathrm{B}$ using $\mathcal{F}_{\mathrm{HCOM}}$ after he sends his input correction value $e$, so we can equivocate it to hit the correct output $z$. This, together with the fact that the outputs of the hash function appear random, is why it is secure to perform the VerLeak step before learning the input of $B$.

As argued above, our modified protocol can be proven secure in the non-extractable, non-programmable random oracle model following the proof of [33]. As we do not require programmability of the random oracle we conjecture that our protocol can be proven secure in the standard (OT hybrid) model using the recently proposed ICE framework of [31], an extension of the UCE framework of [9]. However, we note that it does not seem like our scheme can be proven secure using the UCE framework as in our setting all the garbled gates are related (all garbled with the same $\Delta$ ) and therefore a single leakage phase as prescribed in the UCE framework seems insufficient.

\section{IMPLEMENTATION}

In this section we highlight some of the more technical details of our implementations of the XOR-homomorphic commitment scheme and our final 2PC protocol supporting independent preprocessing. The source code of the project can be found at https://github.com/AarhusCrypto/TinyLEGO.

\section{A. UC-Secure XOR-Homomorphic Commitments}

As part of our full $2 \mathrm{PC}$ prototype we implement the XOR-homomorphic commitment scheme of [34] as a separate subprotocol. This is to our knowledge the first time a scheme following this OT + PRG blueprint has been implemented and we believe our experimental findings are of independent interest. At a high level, the scheme works by the parties initially doing $\tilde{n}$ BaseOTs of security parameter $k$-bit strings, where $\tilde{n}$ is the code-length for some linear error correcting code $\mathcal{C}$ with parameters $[\tilde{n}, \kappa, s]_{\mathbb{F}_{2}}$ with $\kappa$ being the bitlength of the committed messages. The parties then expand the received $k$-bit strings into bit-strings of length $\gamma$ using a PRG which then define the $\gamma$ random commitments the sender is committing to. Next, the sender sends a correctional value for each produced commitment to turn these into codewords of $\mathcal{C}$. Finally, to ensure that the sender sent valid corrections, the receiver challenges the sender to decommit to $2 s$ random linear combinations of all produced commitments. This is done in a way such that no information is leaked about the $\gamma$ committed values. Additively homomorphism then follows from the fact that the code $\mathcal{C}$ is linear and all operations on the expanded PRG strings are linear as well. We highlight the fact that any XOR homomorphic commitment scheme supports the notion of batch opening/decommitment which is similar in nature to the above consistency check. The idea is that the sender initially sends the decommited values directly to the receiver, who hereafter challenges the sender to decommit to $s$ linear combinations of the postulated values where $s$ is the statistical security parameter. Notice that it is only in the initial commit step that $2 s$ combinations are necessary. If the decommitted values match the linear combinations of the postulated values, the receiver accepts. As now only $s$ values are decommitted this approach has the benefit of making the communication overhead independent of the number of values decommitted to. For the full details we refer to [34].

We implement the above scheme in $\mathrm{C}++14$ taking advantage of multi-core capabilities and Intel SSE instructions. We can therefore base the PRG on AES-NI in counter mode and for the error correcting code we use a modified version of the linux kernel implementation of the $\mathrm{BCH}$ code family [17], [38]. As part of the commitment step the parties are required to transpose a binary matrix $S \in\{0,1\}^{\tilde{n} \times \gamma}$ in order to efficiently address the committed values in column-major order. As $\gamma$ in our case can be huge ( $>220$ Mio. for 2000 AES-128 computations) we use the efficient implementation of Ekhlund's cache-efficient algorithm for binary matrix transposition [30] presented in [5], [6]. ${ }^{2}$ As a side note we also augment the OT extension code to support the randomness extraction technique described in Section III-A to implement the $\mathcal{F}_{\Delta \text {-ROT }}$ functionality needed in our $2 \mathrm{PC}$ protocol.

During the development of our implementation we identified the main computational bottleneck of the scheme to be the computation of the random linear combinations. Even if these operations are based on mere XORs, when implemented naively, the number of required instructions is still $\gamma s$ in expectation. Therefore, inspired by [21], we use a different approach for computing the consistency checks using Galois field multiplication. Combined with efficient matrix transposition the effect of using $\mathrm{GF}\left(2^{l}\right)$ multiplication can be seen as computing $l$ linear combinations in parallel. For our particular setting we set $l=128$ as this is the smallest power of 2 greater than the required $2 s$ for $s=40$. We can then use the Intel SSE instruction PCLMULQDQ to very efficiently

\footnotetext{
${ }^{2}$ Available at https://github.com/encryptogroup/OTExtension
} 


\begin{tabular}{|c|c|c|c|c|}
\hline$\gamma$ & \#Threads & \multicolumn{2}{|c|}{ Commit [us] } & Decommit [us] \\
\hline 500 & 1 & 7.21 & $(2815.28)$ & 2.20 \\
\hline 1000 & 2 & 3.85 & (1401.83) & 1.40 \\
\hline 15000 & 4 & 0.64 & (93.99) & 0.34 \\
\hline 50000 & 8 & 0.57 & (28.49) & 0.22 \\
\hline 500000 & 20 & 0.45 & (3.25) & 0.17 \\
\hline 10000000 & 200 & 0.21 & $(0.35)$ & 0.14 \\
\hline 200000000 & 400 & 0.20 & $(0.21)$ & 0.14 \\
\hline
\end{tabular}

TABLE III. TIMINGS FOR COMMITTING TO $\gamma$ STRINGS OF LENGTH 128 BIT WITH $s=40$. ALL TIMINGS ARE $\mu$ S PER COMMITMENT. THE COMMIT TIME IN PARENTHESES INCLUDES THE COST OF THE INITIAL BASEOTS.

compute the $\operatorname{GF}\left(2^{128}\right)$ multiplications. In detail, our approach to compute the checks is as follows:

1) Given a random challenge element $\alpha \in_{R} \operatorname{GF}\left(2^{l}\right)$ the matrix $S$ of committed values in column-major order is split into $u=\lceil S / l\rceil$ blocks $B_{i} \in\{0,1\}^{\tilde{n} \times l}$. Each block is then transposed into row-major order.

2) For $i \in[u]$ and $j \in[\tilde{n}]$, the new matrix $B_{i}^{\prime}=B_{i}^{j} \cdot \alpha^{i}$ is computed where $B_{i}^{j} \in\{0,1\}^{l}$ is the $j$ 'th row of $B_{i}$ interpreted as an element of $\mathrm{GF}\left(2^{l}\right)$.

3) Finally, the combined matrix $B^{\prime}=\sum_{i=1}^{u} B_{i}^{\prime}$ is produced and transposed back into column-major order.

Each column of $B^{\prime}$ can now be seen as a random linear combination of all values of $S$. As a further optimization we see that most GF elements are only multiplied a single time in the above and we can therefore postpone the expensive degree reduction step of the multiplication until $B^{\prime}$ has been fully computed. This is different for computing $\alpha^{i}$ which we therefore reduce at each iteration. In total the required number of degree reductions becomes $n+u$ as opposed to $(n+1) u$. Our experiments show that using the above method of computing 128 linear combinations compared to the naive approach is between 10-13x faster starting at a moderate number of random commitments $\gamma>8000$.

As our implementation of the XOR-homomorphic commitment scheme might be of independent interest we here present our observed timings for committing and decommitting to $\gamma$ random bit-strings of length 128 with $k=128$ and $s=40$. We instantiate the binary $\mathrm{BCH}$ code with parameters $[312,128,41]$ and for convenience we use the implementation of [6] augmented with our randomness extraction technique to compute the required 312 Random OTs. In total this takes about $1400 \mathrm{~ms}$ with our implementation, where $1392 \mathrm{~ms}$ are due to the BaseOTs (using PVW [63]). From the timings reported in [23] we predict that this initial setup step can be done much faster (around $20 \mathrm{~ms}$ ) using their implementation, but since this requires a programmable random oracle assumption and this cost amortizes away as $\gamma$ grows we did not pursue this. Also, if the commitment scheme is used in an application that already relies on oblivious transfer, OT extension can be used to produce the starting BaseOTs at very low cost. We report our findings in Table III. As the scheme requires $\tilde{n}$ BaseOTs to setup we include this cost in the commit timings in parentheses. It can thus be seen by comparing the commitment numbers how the initial OT cost amortizes away as $\gamma$ increases. As there is no initial cost associated with decommitment these timings are only affected by the number of worker-threads we spawn. Furthermore, these experiments were performed on the local LAN setup described in Section VI and not on the Amazon Web Services (AWS) architecture.

\section{B. 2PC with preprocessing using LEGO}

We implement the TinyLEGO protocol with our modifications on top of the previously described commitment scheme. The code is also written in $\mathrm{C}++14$ and makes heavy use of parallelism and Intel SSE instructions for garbling and evaluation of the garbled gates. At a high level, the Generate step is implemented by first partitioning the inputs $(q, n, m)$ into $t$ equally sized subsets for some parameter $t$. The main thread then starts $t$ parallel executions of the generate step with two synchronization points, one where the commitment to $\Delta$ is sent (which only one execution is charged with), and one after the cut-and-choose step. The latter is necessary as the random permutation that describes the initial bucketing must only be revealed after all garbled components have been sent to $B$. We emphasize that it is due to our preprocessing being independent of the structure of $f$ that we can trivially parallelize the above step using any number of threads $t$. Due to the above design we also run $t$ executions of the commitment scheme, however for the PRG expansion we use the same seed OT values in all executions. As the PRG is based on a block cipher in counter mode this is not an issue as execution $i+1$ sets it's counter sufficiently high compared to the $i$ 'th execution so there is no overlap. Since they all use the same seed OTs the choice-bits are also the same across all executions and they can therefore be combined in the same way as for a single execution.

The Build and Eval phases follow roughly the same design pattern as above. We note however that in these phases each thread is responsible for soldering and evaluating an entire circuit. The garbling and evaluation of garbled gates and wire authenticators are implemented purely as 128-bit SSE instructions to maximize performance. We base the hash function for garbling gates and producing wire authenticators on Fixed-Key AES-NI as advocated in [10]. This choice is mainly motivated by producing as comparable results as possible to previous works that are also based on Fixed-Key AES-NI.

The Eval phase consists of two rounds, one where $B$ specifies the input mask and one where $A$ replies with its keys and decommitments. A's reply has communication complexity $k n_{\mathrm{A}}$ for A's input keys and $(\tilde{n}+k)\left(n_{\mathrm{B}}+m\right)$ for the decommitments of B's input keys and the lsb masks of the output keys, where $\tilde{n}$ is the code-length of the $\mathrm{BCH}$ code. We note that the communication cost of the decommitments can be reduced to $(\tilde{n}+k) s+k\left(n_{\mathrm{B}}+m\right)$ using the batch decommit approach mentioned in Section V-A, but at the cost of adding an additional round. For the circuits used in our experiments (AES-128, SHA-256) we observed a loss of around a factor 1.25 in the LAN setting and much more in the WAN setting with this approach. Still, for other circuits where the ratio $\left(n_{\mathrm{B}}+m\right) /|f|$ is substantial and both network latency and bandwidth are low we suspect that adding this extra round can pay off. Finally if one is willing to assume a programmable random oracle the online cost for the output bits can be eliminated entirely as the simulator then can program the oracle to output matching output keys for a preprocessed decommitment lsb-bit once it learns the final output. 


\section{PERFORMANCE}

To give a broad view of the performance of our prototype we run experiments in a local LAN setting and on both a LAN and WAN on the Amazon Web Services (AWS). In more detail:

Local LAN with two machines, one acting as A and the other acting as B. We measured a total bandwidth of $942 \mathrm{Mbits} / \mathrm{sec}$ with round trip time (rtt) $0.12 \mathrm{~ms}$. Both machines run Ubuntu 16.04 with an Intel Ivy Bridge i7 $3.5 \mathrm{GHz}$ quad-core processor and $32 \mathrm{~GB}$ DDR3 RAM.

AWS LAN with two c4.8xlarge instances located in the Virginia region connected via a high performance LAN. We measured a bandwidth of 9.52 Gbits/sec with rtt $0.16 \mathrm{~ms}$. Both machines run Amazon Linux AMI 2016.03.2 with an Intel Xeon E5-2666 v3 (Haswell) processor with 36 vCPUs and 60 GB RAM.

AWS WAN with two c4.8xlarge instances, one in Virginia and one in Ireland. We measured a bandwidth of 214 Mbits/sec on average for a single TCP connection and up to $3.17 \mathrm{Gbit} / \mathrm{sec}$ when running many parallel connections. The rtt measured was $81.32 \mathrm{~ms}$. Both machines run Amazon Linux AMI 2016.03.2 with an Intel Xeon E5-2666 v3 (Haswell) processor with 36 vCPUs and 60 GB RAM.

For all settings the code was compiled using GCC-5.4 with the -O3 optimization flag set. As mentioned in Section V-A the implementation used for the BaseOTs are based on [6] using PVW [63]. If one is willing to assume a programmable random oracle, these can be replaced with the fast protocol and implementation of [23] and we would expect a total cost around $20 \mathrm{~ms}$ as opposed to $850 \mathrm{~ms}$ (AWS LAN) with the current implementation.

\section{A. Our Performance Results}

We summarize our measured results in Table IV on the following page for the three above-mentioned settings. All numbers reported are averages of 10 executions. Not surprisingly we see the best performance on the AWS machines in the LAN setting where we can evaluate an AES-128 circuit with latency $1.13 \mathrm{~ms}$ or $0.08 \mathrm{~ms}$ throughput per AES-128 in the online phase. We also see that when considering 1024 AES128 evaluations the dependent preprocessing + the online phase is below $2 \mathrm{~ms}$. When including the cost of the independent preprocessing each AES-128 can be done in total time less than $16 \mathrm{~ms}$. Similarly when considering 256 SHA-256 evaluations the online phase can be done with latency $9.14 \mathrm{~ms}$ or $1.05 \mathrm{~ms}$ of throughput per SHA-256. Also the dependent preprocessing + online phase and total cost is below $22 \mathrm{~ms}$ and $205 \mathrm{~ms}$, respectively (when preprocessing material enough for 256 SHA-256 evaluations).

For the single execution setting we see a significant increase in execution time for the dependent preprocessing compared to above. This is due to the design of our prototype which only uses multiple execution threads in the dependent preprocessing and online phases if several, possibly different, circuits are processed at the same time. We also note that our prototype requires a large amount of RAM as we store all garbling material and commitments in-memory. This design choice is due to convenience, but for a deployed system based on the LEGO approach this should be addressed using external memory sources with support for pipelined evaluation as described in Section IV.

For the AWS WAN setting we see that a single execution of AES-128 takes around $83 \mathrm{~ms}$ online time where almost all of the runtime is spent waiting due to a latency of $\sim 81 \mathrm{~ms}$. The latency also severely impacts the two preprocessing phases where the independent preprocessing takes around $1882 \mathrm{~ms}$ (20x compared to AWS LAN) and the dependent offline phase takes $96 \mathrm{~ms}$ ( $7 \mathrm{x}$ compared to AWS LAN). However this overhead can be somewhat mitigated when considering several circuits, down to a factor $2-4 \mathrm{x}$ compared to AWS LAN due to the computation and communication being more interleaved and better utilization of the bandwidth with several TCP connections.

Finally in Table $\mathrm{V}$ on the next page we report on the amount of data our prototype transfers from the circuit constructor to the circuit evaluator for both AES-128 and SHA-256. For clarity we have not included the communication from evaluator to constructor, but note that for 1024 AES-128 and 256 SHA-256 a total of $8.12 \mathrm{MB}$ and $4.09 \mathrm{MB}$ are transferred, respectively, and for both cases around $99 \%$ of the communication stems from the initial BaseOTs. The table also summarizes the bucketing parameters used in our experiments, which have been chosen so that the probability bound given by Theorem 2 in Section IV-A is negligible. Also we set the two input bucket parameters $\tilde{\beta}=2 \beta+1$ and $\tilde{\alpha}=2 \alpha+1$ which ensures a correct majority for all the input buckets and authenticators except with negligible probability. For the data numbers in Table $\mathrm{V}$ it can be seen in parentheses how the relative preprocessing cost of a circuit decreases as more evaluations are considered. We highlight that in this work (and previous LEGO protocols) this is due to the increasing number of gates produced, not by the number of circuits. As an example of this effect, going from a single AES-128 with 6928 gates $^{3}$ to 1024 AES-128 with 7094272 gates decreases the cost of the independent preprocessing by a factor $2.3 \mathrm{x}$ per AES-128, from $14.94 \mathrm{MB}$ to $6.42 \mathrm{MB}$. It is worth noting that the "LEGO effect" only applies to the independent preprocessing. This is because in the subsequent dependent preprocessing step two solderings ( $k$ bits each) are sent per gate of the circuit $f$ and not for each garbled gate produced. In addition a small constant $2.2 \mathrm{kB}$ of decommitment data is transferred in this phase for the $s$ challenge linear combinations. For the online step the communication consists of $n_{\mathrm{A}} k$ bits for the constructors input $+(\tilde{n}+k)\left(n_{\mathrm{B}}+m\right)$ bits for the decommitments to the evaluators input and the output decoding bits where $\tilde{n}$ is the code-length of the ECC $\mathcal{C}$ used in the commitment scheme. In Section V-B we discussed how this could further be reduced to $(\tilde{n}+k) s+k\left(n_{\mathrm{B}}+m\right)$ at the price of adding an extra round to the online phase.

\section{B. Comparison with Related Work}

We compare our measured timings to those reported in the recent works of [56] and [65], both of which are solely applicable in the amortized setting. In contrast our protocol can naturally handle the single execution setting, along with a more general amortized setting where several distinct functions

\footnotetext{
${ }^{3}$ We use the AES non-expanded circuit of [68] which has 6800 AND gates. However we augment the circuit with identity gates on the 128 output wires in order to simplify output decoding using VerLeak.
} 


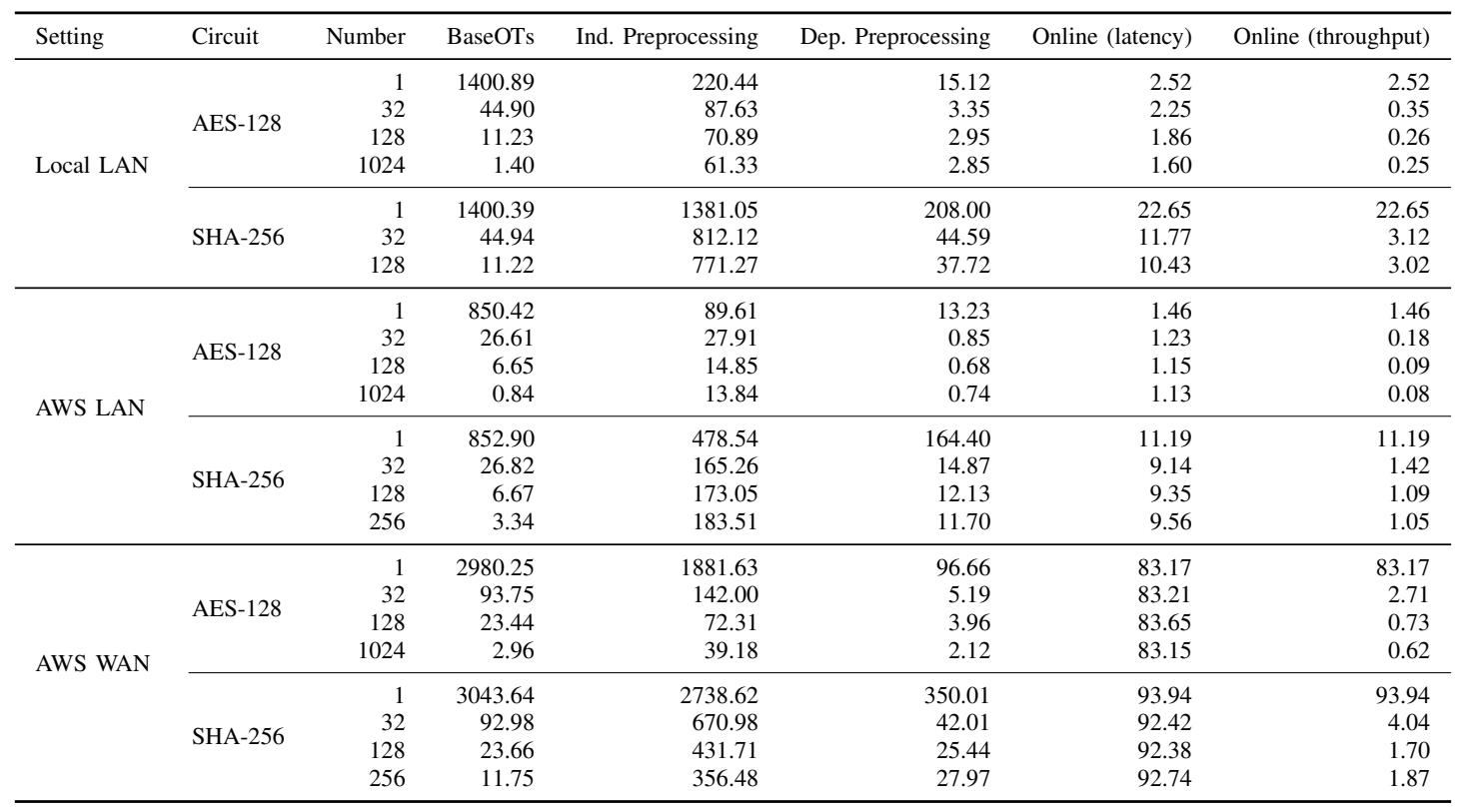

TABLE IV. EVALUATOR TIMINGS MEASURED IN THE LOCAL LAN, AWS LAN AND AWS WAN SETTING FOR AES-128 AND SHA-256 WITH $k=128$ AND $s=40$. ALL TIMINGS ARE ms PER CIRCUIT.

\begin{tabular}{|c|c|c|c|c|c|c|c|c|c|c|c|c|c|}
\hline Circuit & Number & $\beta$ & $p_{g}$ & $\alpha$ & $p_{a}$ & Base & & Ind. Pre & ocessing & Dep. Pr & rocessing & & \\
\hline \multirow{4}{*}{ AES-128 } & 1 & 7 & $2^{-4}$ & 6 & $2^{-3}$ & $19.52 \mathrm{kB}$ & & $14.94 \mathrm{MB}$ & & $226.86 \mathrm{kB}$ & & $16.13 \mathrm{kB}$ & \\
\hline & 32 & 4 & $2^{-2}$ & 3 & $2^{-2}$ & $19.52 \mathrm{kB}$ & $(610 \mathrm{~B})$ & $279.78 \mathrm{MB}$ & $(8.74 \mathrm{MB})$ & $7.26 \mathrm{MB}$ & $(226.86 \mathrm{kB})$ & $516.10 \mathrm{kB}$ & $(16.13 \mathrm{kB})$ \\
\hline & 128 & 4 & $2^{-3}$ & 3 & $2^{-3}$ & $19.52 \mathrm{kB}$ & (153 B) & $924.68 \mathrm{MB}$ & $(7.22 \mathrm{MB})$ & $29.04 \mathrm{MB}$ & $(226.86 \mathrm{kB})$ & $2.06 \mathrm{MB}$ & $(16.13 \mathrm{kB})$ \\
\hline & 1024 & 4 & $2^{-5}$ & 3 & $2^{-6}$ & $19.52 \mathrm{kB}$ & (19 B) & $6.57 \mathrm{~GB}$ & $(6.42 \mathrm{MB})$ & $232.31 \mathrm{MB}$ & $(226.86 \mathrm{kB})$ & $16.52 \mathrm{MB}$ & $(16.13 \mathrm{kB})$ \\
\hline \multirow{4}{*}{ SHA-256 } & 1 & 5 & $2^{-3}$ & 4 & $2^{-4}$ & $19.52 \mathrm{kB}$ & & $120.34 \mathrm{MB}$ & & $2.93 \mathrm{MB}$ & & $22.23 \mathrm{kB}$ & \\
\hline & 32 & 4 & $2^{-4}$ & 3 & $2^{-5}$ & $19.52 \mathrm{kB}$ & $(610 \mathrm{~B})$ & $2.73 \mathrm{~GB}$ & (85.19 MB) & $93.63 \mathrm{MB}$ & $(2.93 \mathrm{MB})$ & $712.70 \mathrm{kB}$ & $(22.23 \mathrm{kB})$ \\
\hline & 128 & 4 & $2^{-6}$ & 3 & $2^{-5}$ & $19.52 \mathrm{kB}$ & (153 B) & $10.31 \mathrm{~GB}$ & (80.54 MB) & $374.52 \mathrm{MB}$ & $(2.93 \mathrm{MB})$ & $2.85 \mathrm{MB}$ & $(22.23 \mathrm{kB})$ \\
\hline & 256 & 4 & $2^{-7}$ & 3 & $2^{-5}$ & $19.52 \mathrm{kB}$ & $(76 \mathrm{~B})$ & $20.28 \mathrm{~GB}$ & $(79.20 \mathrm{MB})$ & $749.03 \mathrm{MB}$ & $(2.93 \mathrm{MB})$ & $5.70 \mathrm{MB}$ & $(22.23 \mathrm{kB})$ \\
\hline
\end{tabular}

TABLE V. BUCKETING PARAMETERS AND DATA RECEIVED BY THE EVALUATOR IN THE DIFFERENT PHASES OF OUR PROTOCOL FOR AES-128 AND SHA-256 WITH $k=128$ AND $s=40$. NUMBERS IN PARENTHESES ARE DATA PER CIRCUIT PRODUCED.

\begin{tabular}{|c|c|c|c|c|c|}
\hline Protocol & Setting & Number & Ind. Preprocessing & Offline & Online \\
\hline \multirow{6}{*}{ [56] } & \multirow{3}{*}{ AWS LAN } & 32 & $x$ & 197 & 12 \\
\hline & & 128 & $x$ & 114 & 10 \\
\hline & & 1024 & $x$ & 74 & 7 \\
\hline & \multirow{3}{*}{ AWS WAN } & 32 & $x$ & 1126 & 163 \\
\hline & & 128 & $x$ & 919 & 164 \\
\hline & & 1024 & $x$ & 759 & 160 \\
\hline \multirow{6}{*}{ [65] } & \multirow{3}{*}{ AWS LAN } & 32 & $x$ & 45 & 1.7 \\
\hline & & 128 & $x$ & 16 & 1.5 \\
\hline & & 1024 & $x$ & 5.1 & 1.3 \\
\hline & \multirow{3}{*}{ AWS WAN } & 32 & $x$ & 282 & 190 \\
\hline & & 128 & $x$ & 71 & 191 \\
\hline & & 1024 & $x$ & 34 & 189 \\
\hline \multirow{6}{*}{ This Work } & \multirow{3}{*}{ AWS LAN } & 32 & 54.52 & 0.85 & 1.23 \\
\hline & & 128 & 21.5 & 0.68 & 1.15 \\
\hline & & 1024 & 14.68 & 0.74 & 1.13 \\
\hline & \multirow{3}{*}{ AWS WAN } & 32 & 235.75 & 5.19 & 83.21 \\
\hline & & 128 & 95.75 & 3.96 & 83.65 \\
\hline & & 1024 & 42.14 & 2.12 & 83.15 \\
\hline
\end{tabular}

TABLE VI. COMPARISON OF THE REPORTED TIMINGS FOR AES-128 IN THE AWS LAN AND AWS WAN SETTING WITH $k=128$ AND $s=40$. THE PREPROCESSING COLUMN INCLUDES THE COST OF THE BASEOTS FOR THIS WORK. All TIMINGS ARE ms PER AES-128. BEST RESUlTS MARKED IN BOLD. can be preprocessed in the same batch. However to make a meaningful comparison we focus on the "traditional" amortized setting considering 32, 128, and 1024 AES-128 computations and we summarize the comparison in Table VI. The independent preprocessing timings for our protocol consists of the BaseOTs + the independent preprocessing. The first thing to notice is that for applications where independent preprocessing is applicable, and can therefore be disregarded, our dependent offline performance is superior to both prior works for any number of AES-128 computations by a large margin. Compared to [56] our reported timings are better by $100-358 x$ depending on the setting and number of circuits. For [65] the gap is smaller, but still substantial, namely by $6-54 \mathrm{x}$. For applications where independent preprocessing can not be utilized we are still superior to the work of [56], but for most settings and number of circuits we cannot compete with the offline phase of [65]. However the differences are typically within a factor 1.2-3x.

For the online timings in the AWS LAN setting for AES-128 we measure faster overall timings than [65] for all number of circuits by a tiny margin. As the differences are less than half a millisecond we believe the only reasonable thing to conclude is that the online times are comparable. Though 


\begin{tabular}{lccrr}
\hline Protocol & Number & Ind. Preprocessing & Offline & Online \\
\hline \multirow{4}{*}[56]{} & 32 & $\boldsymbol{x}$ & $8.13 \mathrm{MB}$ & $312 \mathrm{kB}$ \\
& 128 & $\boldsymbol{x}$ & $5.45 \mathrm{MB}$ & $238 \mathrm{kB}$ \\
& 1024 & $\boldsymbol{x}$ & $3.76 \mathrm{MB}$ & $170 \mathrm{kB}$ \\
\hline \multirow{3}{*}[65]{$^{*}$} & 32 & $\boldsymbol{x}$ & $3.75 \mathrm{MB}$ & $25.76 \mathrm{kB}$ \\
& 128 & $\boldsymbol{x}$ & $2.5 \mathrm{MB}$ & $21.31 \mathrm{kB}$ \\
& 1024 & $\boldsymbol{x}$ & $1.56 \mathrm{MB}$ & $16.95 \mathrm{kB}$ \\
\hline \multirow{3}{*}{ This Work } & 32 & $8.74 \mathrm{MB}$ & $\mathbf{2 2 6 . 8 6} \mathbf{~ k B}$ & $\mathbf{1 6 . 1 3} \mathbf{~ k B}$ \\
& 128 & $7.22 \mathrm{MB}$ & $\mathbf{2 2 6 . 8 6} \mathbf{~ k B}$ & $\mathbf{1 6 . 1 3} \mathbf{~ k B}$ \\
& 1024 & $6.42 \mathrm{MB}$ & $\mathbf{2 2 6 . 8 6} \mathbf{~ k B}$ & $\mathbf{1 6 . 1 3} \mathbf{~ k B}$ \\
\hline
\end{tabular}

TABLE VII. COMPARISON OF THE DATA RECEIVED BY THE EVALUATOR FOR DIFFERENT NUMBER OF EXECUTIONS OF AES-128 WITH $k=128$ AND $s=40$. ALL NUMBERS ARE DATA PER AES-128. BEST RESULTS MARKED IN BOLD.

when looking at raw throughput we outperform [65] by more than a factor $3 \mathrm{x}(0.08 \mathrm{~ms}$ vs. $0.26 \mathrm{~ms})$. Going beyond Table VI and considering the larger SHA-256 circuit we note that our online phase is not faster than [65] $(9.35 \mathrm{~ms}$ vs. $8.8 \mathrm{~ms}$ for 128 circuits). This is again due to the design of our prototype that uses a single execution thread in the online phase per circuit, while [65] uses several threads. We therefore see it as an interesting problem for future research to tailor the LEGO online phase to better exploit parallelism for a single circuit.

With regards to online latency in the AWS WAN setting there is however no doubt that our two round online phase outperforms both [56] and [65]. This is directly related to the previous protocols having more rounds which in a high latency network significantly decreases performance. For comparison [56] has a 4 round online phase and [65] has 5. One thing to note however is that [65] delivers output to both parties in 5 rounds, whereas both our work and [56] would need an extra round to support this. Also the implementation of [56] is written in a mix of Java and C++ using JNI which definitely adds overhead to the running time. It is however unclear how much of a speedup a native implementation would achieve, but we suspect it would be substantial. The implementation of [65] is written solely in $\mathrm{C}++$ and according to the paper also takes full advantage of parallelization.

In Table VII we summarize the required communication for the previously considered protocols and our work for the same setting as Table VI. As was the case for the measured timings, when disregarding the cost of the independent preprocessing, our protocol requires significantly less communication in both the offline and online phase compared to the previous works. For the offline phase the communication is 5-12x less than [65] and 16-358x less than [56]. If the independent preprocessing is included as part of the offline phase our protocol however requires transferring more raw data than the previous two works for any of the considered number of circuits. However this is only so because we are considering multiple copies (32, 128, and 1024) of the same function AES-128. If we instead consider settings with few copies (the larger the better), a single copy, or several different circuits, the amount of data received in the independent + dependent preprocessing phase of our protocol can match or be lower than the dependent offline phase of [65], depending on the circuit sizes and number of circuits considered. Also, as [65] uses the dual-execution paradigm where both parties send and receive the same amount of data, the above comparison is only meaningful assuming a full-duplex channel which might not always be available. Finally, even if the amount of data received by the evaluator in our protocol is up to $\sim 4 \mathrm{x}$ that of [65] in Table VII, due to the highly parallelizable nature of our independent preprocessing phase, this does not translate into equivalently lower performance as can be seen in Table VI.

For the online phase, our protocol is more data-efficient than the previous works for any of the considered settings. In particular, we require sending $16.13 \mathrm{kB}$ per $\mathrm{AES}$ which is around 1.05-1.6x less data than [65] and 10.5-19x less than [56], depending on the number of executions considered. Furthermore if one is willing to assume a programmable random oracle, as is already the case of both [65] and [56], our online phase can easily be modified to only sending $6.30 \mathrm{kB}$ (using 3 rounds) or $9.09 \mathrm{kB}$ (using 2 rounds) as explained in Section V-B.

Finally as mentioned in Section I and summarized in Table I the best reported timings for evaluating a single AES-128 is $65 \mathrm{~ms}$ in [69]. Based on the reported numbers in their paper we estimate that $\sim 20 \mathrm{~ms}$ of the execution time consists of the initial BaseOTs. We therefore consider the actual cost of their protocol to be around $45 \mathrm{~ms}$ and motivate this by observing that a single computation of BaseOTs can be reused for any number of future executions using OT extension. To give as meaningful a comparison as possible we also ran our implementation on the same AWS setup (c4.2x instance) for the single execution AES-128 case measuring $105.7 \mathrm{~ms}$ of ind. preprocessing time, $12.07 \mathrm{~ms}$ dep. preprocessing time and $1.41 \mathrm{~ms}$ online time on a LAN. Therefore our protocol performs around $2.5 \mathrm{x}$ times slower than theirs in total time (when also ignoring the cost of our initial BaseOTs). However if ind. preprocessing can be applied, then from the time the circuit is input by the parties, our protocol takes around $13.48 \mathrm{~ms}$ to evaluate, which is around $3.5 \mathrm{x}$ faster than [69]. We ran the same experiment in the WAN setting where we evaluate an AES-128 in $1837 \mathrm{~ms}$ of ind. preprocessing time, $82.51 \mathrm{~ms}$ dep. preprocessing time and $72.63 \mathrm{~ms}$ online time. As [69] takes $1513 \mathrm{~ms}$ in total, when adjusting for initial BaseOT cost the difference is about a factor $1.5 \mathrm{x}$ in favor of the latter. However, when ignoring time for independent preprocessing our protocol can perform around an order of magnitude faster. We believe this difference in factors between LAN and WAN is due to our protocol having fewer rounds (when ignoring our preprocessing) and our implementation fully saturating the network as it sets up multiple parallel TCP connections for maximal bandwidth utilization.

\section{ACKNOWLEDGMENTS}

The authors would like to thank Michael Zohner for his assistance in augmenting the code of [6] to support the randomness extraction technique used to produce $\Delta$-ROTs. We also thank Tore Kasper Frederiksen and Thomas P. Jakobsen for sharing their initial unpublished work on implementing TinyLEGO which was used as inspiration for part of our codebase. Finally, we thank the anonymous reviewers from NDSS 2017 for their valuable feedback on our paper. 


\section{REFERENCES}

[1] Dyadic Security. https://www.dyadicsec.com.

[2] Partisia. http://www.partisia.dk.

[3] Sepior. https://www.sepior.com.

[4] A. Afshar, P. Mohassel, B. Pinkas, and B. Riva, "Non-interactive secure computation based on cut-and-choose," in EUROCRYPT 2014, ser LNCS, P. Q. Nguyen and E. Oswald, Eds., vol. 8441. Springer, May 2014, pp. 387-404.

[5] G. Asharov, Y. Lindell, T. Schneider, and M. Zohner, "More efficient oblivious transfer and extensions for faster secure computation," in $A C M$ CCS 2013, A.-R. Sadeghi, V. D. Gligor, and M. Yung, Eds. ACM Press, Nov. 2013, pp. 535-548.

[6] - "More efficient oblivious transfer extensions with security for malicious adversaries," in EUROCRYPT 2015, Part I, ser. LNCS, E. Oswald and M. Fischlin, Eds., vol. 9056. Springer, Apr. 2015, pp. 673-701

[7] Y. Aumann and Y. Lindell, "Security against covert adversaries: Efficient protocols for realistic adversaries," in TCC 2007, ser. LNCS, S. P. Vadhan, Ed., vol. 4392. Springer, Feb. 2007, pp. 137-156.

[8] D. Beaver, "Precomputing oblivious transfer," in CRYPTO 1995, ser LNCS, D. Coppersmith, Ed., vol. $963 . \quad$ Springer, Aug. 1995, pp. $97-$ 109.

[9] M. Bellare, V. T. Hoang, and S. Keelveedhi, "Instantiating random oracles via UCEs," in CRYPTO 2013, Part II, ser. LNCS, R. Canetti and J. A. Garay, Eds., vol. 8043. Springer, Aug. 2013, pp. 398-415.

[10] M. Bellare, V. T. Hoang, S. Keelveedhi, and P. Rogaway, "Efficient garbling from a fixed-key blockcipher," in IEEE S\&P 2013. IEEE Computer Society Press, May 2013, pp. 478-492.

[11] M. Bellare, V. T. Hoang, and P. Rogaway, "Adaptively secure garbling with applications to one-time programs and secure outsourcing," in ASIACRYPT 2012, ser. LNCS, X. Wang and K. Sako, Eds., vol. 7658. Springer, Dec. 2012, pp. 134-153.

[12] - "Foundations of garbled circuits," in ACM CCS 2012, T. Yu, G. Danezis, and V. D. Gligor, Eds. ACM Press, Oct. 2012, pp. 784 796.

[13] M. Bellare and P. Rogaway, "Random oracles are practical: A paradigm for designing efficient protocols," in ACM CCS 1993, V. Ashby, Ed ACM Press, Nov. 1993, pp. 62-73.

[14] M. Ben-Or, S. Goldwasser, and A. Wigderson, "Completeness theorems for non-cryptographic fault-tolerant distributed computation (extended abstract)," in STOC 1988. ACM Press, May 1988, pp. 1-10.

[15] D. Bogdanov, S. Laur, and J. Willemson, "Sharemind: A framework for fast privacy-preserving computations," in ESORICS 2008, ser. LNCS S. Jajodia and J. López, Eds., vol. 5283. Springer, Oct. 2008, pp. 192-206.

[16] P. Bogetoft, D. L. Christensen, I. Damgård, M. Geisler, T. Jakobsen, M. Krøigaard, J. D. Nielsen, J. B. Nielsen, K. Nielsen, J. Pagter, M. I. Schwartzbach, and T. Toft, "Secure multiparty computation goes live," in FC 2009, ser. LNCS, R. Dingledine and P. Golle, Eds., vol. 5628. Springer, Feb. 2009, pp. 325-343.

[17] R. C. Bose and D. K. Ray-Chaudhuri, "On a class of error correcting binary group codes," Information and control, vol. 3, no. 1, pp. 68-79, 1960

[18] L. T. A. N. Brandão, "Secure two-party computation with reusable bit-commitments, via a cut-and-choose with forge-and-lose technique (extended abstract)," in ASIACRYPT 2013, Part II, ser. LNCS, K. Sako and P. Sarkar, Eds., vol. 8270. Springer, Dec. 2013, pp. 441-463.

[19] S. S. Burra, E. Larraia, J. B. Nielsen, P. S. Nordholt, C. Orlandi, E. Orsini, P. Scholl, and N. P. Smart, "High performance multi-party computation for binary circuits based on oblivious transfer," IACR Cryptology ePrint Archive, vol. 2015, p. 472, 2015. [Online]. Available: http://eprint.iacr.org/2015/472

[20] R. Canetti, "Universally composable security: A new paradigm for cryptographic protocols," in FOCS 2001. IEEE Computer Society Press, Oct. 2001, pp. 136-145.

[21] I. Cascudo, I. Damgård, B. David, N. Döttling, and J. B. Nielsen, "Rate-1, linear time and additively homomorphic UC commitments," in CRYPTO 2016, Part III, ser. LNCS, M. Robshaw and J. Katz, Eds., vol. 9816. Springer, 2016, pp. 179-207.
[22] D. Chaum, C. Crépeau, and I. Damgård, "Multiparty unconditionally secure protocols (extended abstract)," in STOC 1988. ACM Press, May 1988, pp. 11-19.

[23] T. Chou and C. Orlandi, "The simplest protocol for oblivious transfer," in LATINCRYPT 2015, ser. LNCS, K. E. Lauter and F. RodríguezHenríquez, Eds., vol. 9230. Springer, Aug. 2015, pp. 40-58.

[24] I. Damgård, M. Keller, E. Larraia, V. Pastro, P. Scholl, and N. P. Smart, "Practical covertly secure MPC for dishonest majority - or: Breaking the SPDZ limits," in ESORICS 2013, ser. LNCS, J. Crampton, S. Jajodia, and K. Mayes, Eds., vol. 8134. Springer, Sep. 2013, pp. 1-18.

[25] I. Damgård, R. Lauritsen, and T. Toft, "An empirical study and some improvements of the MiniMac protocol for secure computation," in SCN 2014, ser. LNCS, M. Abdalla and R. D. Prisco, Eds., vol. 8642. Springer, Sep. 2014, pp. 398-415.

[26] I. Damgård, J. B. Nielsen, M. Nielsen, and S. Ranellucci, "Gatescrambling revisited - or: The TinyTable protocol for 2-party secure computation," IACR Cryptology ePrint Archive, vol. 2016, p. 695, 2016. [Online]. Available: http://eprint.iacr.org/2016/695

[27] I. Damgård, V. Pastro, N. P. Smart, and S. Zakarias, "Multiparty computation from somewhat homomorphic encryption," in CRYPTO 2012, ser. LNCS, R. Safavi-Naini and R. Canetti, Eds., vol. $7417 . \quad$ Springer, Aug. 2012, pp. 643-662.

[28] I. Damgård and R. W. Zakarias, "Fast oblivious AES A dedicated application of the MiniMac protocol," in AFRICACRYPT 2016, ser. LNCS, vol. 9646. Springer, 2016, pp. 245-264.

[29] I. Damgård and S. Zakarias, "Constant-overhead secure computation of boolean circuits using preprocessing," in TCC 2013, ser. LNCS, A. Sahai, Ed., vol. 7785. Springer, Mar. 2013, pp. 621-641.

[30] J. O. Eklundh, "A fast computer method for matrix transposing," IEEE Trans. Computers, vol. 21, no. 7, pp. 801-803, 1972.

[31] P. Farshim and A. Mittelbach, "Modeling random oracles under unpredictable queries," in FSE 2016, ser. LNCS, T. Peyrin, Ed., vol. 9783 Springer, March, 2016, pp. 453-473.

[32] T. K. Frederiksen, T. P. Jakobsen, and J. B. Nielsen, "Faster maliciously secure two-party computation using the GPU," in SCN 2014, ser. LNCS, M. Abdalla and R. D. Prisco, Eds., vol. 8642. Springer, Sep. 2014, pp. 358-379.

[33] T. K. Frederiksen, T. P. Jakobsen, J. B. Nielsen, and R. Trifiletti, "TinyLEGO: An interactive garbling scheme for maliciously secure two-party computation," IACR Cryptology ePrint Archive, vol. 2015, p. 309, 2015. [Online]. Available: http://eprint.iacr.org/2015/309

[34] _ "On the complexity of additively homomorphic UC commitments," in TCC 2016-A, Part I, ser. LNCS, E. Kushilevitz and T. Malkin, Eds., vol. 9562. Springer, Jan. 2016, pp. 542-565.

[35] T. K. Frederiksen, T. P. Jakobsen, J. B. Nielsen, P. S. Nordholt, and C. Orlandi, "MiniLEGO: Efficient secure two-party computation from general assumptions," in EUROCRYPT 2013, ser. LNCS, T. Johansson and P. Q. Nguyen, Eds., vol. 7881. Springer, May 2013, pp. 537-556.

[36] T. K. Frederiksen and J. B. Nielsen, "Fast and maliciously secure twoparty computation using the GPU," in ACNS 2013, ser. LNCS, M. J. Jacobson Jr., M. E. Locasto, P. Mohassel, and R. Safavi-Naini, Eds., vol. 7954. Springer, Jun. 2013, pp. 339-356.

[37] O. Goldreich, S. Micali, and A. Wigderson, "How to play any mental game or A completeness theorem for protocols with honest majority," in STOC 1987, A. Aho, Ed. ACM Press, May 1987, pp. 218-229.

[38] A. Hocquenghem, "Codes correcteurs d'erreurs," Chiffres, vol. 2, no 147-156, pp. 8-5, 1959.

[39] Y. Huang, D. Evans, J. Katz, and L. Malka, "Faster secure twoparty computation using garbled circuits," in USENIX Security 2011 USENIX Association, 2011.

[40] Y. Huang, J. Katz, and D. Evans, "Efficient secure two-party computation using symmetric cut-and-choose," in CRYPTO 2013, Part II, ser. LNCS, R. Canetti and J. A. Garay, Eds., vol. 8043. Springer, Aug. 2013, pp. 18-35.

[41] Y. Huang and R. Zhu, "Revisiting LEGOs: Optimizations, analysis, and their limit," IACR Cryptology ePrint Archive, vol. 2015, p. 1038, 2015. [Online]. Available: http://eprint.iacr.org/2015/1038

[42] N. Husted, S. Myers, a. shelat, and P. Grubbs, "GPU and CPU parallelization of honest-but-curious secure two-party computation," in ACSAC 2013, C. N. P. Jr., Ed. ACM, 2013, pp. 169-178. 
[43] M. Keller, E. Orsini, and P. Scholl, "Actively secure OT extension with optimal overhead," in CRYPTO 2015, Part I, ser. LNCS, R. Gennaro and M. J. B. Robshaw, Eds., vol. $9215 . \quad$ Springer, Aug. 2015, pp. 724-741.

[44] _ "MASCOT: faster malicious arithmetic secure computation with oblivious transfer," in ACM CCS 2016, E. R. Weippl, S. Katzenbeisser, C. Kruegel, A. C. Myers, and S. Halevi, Eds. ACM Press, Oct. 2016, pp. 830-842.

[45] M. Keller, P. Scholl, and N. P. Smart, "An architecture for practical actively secure MPC with dishonest majority," in ACM CCS 2013, A.R. Sadeghi, V. D. Gligor, and M. Yung, Eds. ACM Press, Nov. 2013, pp. 549-560.

[46] M. S. Kiraz and B. Schoenmakers, "A protocol issue for the malicious case of Yao's garbled circuit construction," in 27th Symposium on Information Theory in the Benelux, 2006, pp. 283-290.

[47] V. Kolesnikov and T. Schneider, "Improved garbled circuit: Free XOR gates and applications," in ICALP 2008, Part II, ser. LNCS, L. Aceto, I. Damgård, L. A. Goldberg, M. M. Halldórsson, A. Ingólfsdóttir, and I. Walukiewicz, Eds., vol. 5126. Springer, Jul. 2008, pp. 486-498.

[48] B. Kreuter, a. shelat, and C. Shen, "Billion-gate secure computation with malicious adversaries," in USENIX Security 2012. USENIX Association, 2012, pp. 285-300.

[49] E. Larraia, E. Orsini, and N. P. Smart, "Dishonest majority multi-party computation for binary circuits," in CRYPTO 2014, Part II, ser. LNCS, J. A. Garay and R. Gennaro, Eds., vol. 8617. Springer, Aug. 2014, pp. $495-512$.

[50] Y. Lindell, "Fast cut-and-choose based protocols for malicious and covert adversaries," in CRYPTO 2013, Part II, ser. LNCS, R. Canetti and J. A. Garay, Eds., vol. 8043. Springer, Aug. 2013, pp. 1-17.

[51] Y. Lindell and B. Pinkas, "An efficient protocol for secure two-party computation in the presence of malicious adversaries," in EUROCRYPT 2007, ser. LNCS, M. Naor, Ed., vol. 4515. Springer, May 2007, pp. 52-78.

[52] - "A proof of security of Yao's protocol for two-party computation," Journal of Cryptology, vol. 22, no. 2, pp. 161-188, Apr. 2009.

[53] — "Secure two-party computation via cut-and-choose oblivious transfer," in TCC 2011, ser. LNCS, Y. Ishai, Ed., vol. 6597. Springer, Mar. 2011, pp. 329-346.

[54] Y. Lindell, B. Pinkas, and N. P. Smart, "Implementing two-party computation efficiently with security against malicious adversaries," in SCN 2008, ser. LNCS, R. Ostrovsky, R. D. Prisco, and I. Visconti, Eds., vol. 5229. Springer, Sep. 2008, pp. 2-20.

[55] Y. Lindell and B. Riva, "Cut-and-choose Yao-based secure computation in the online/offline and batch settings," in CRYPTO 2014, Part II, ser. LNCS, J. A. Garay and R. Gennaro, Eds., vol. 8617. Springer, Aug. 2014, pp. 476-494.

[56] — "Blazing fast $2 \mathrm{PC}$ in the offline/online setting with security for malicious adversaries," in ACM CCS 2015, I. Ray, N. Li, and C. Kruegel:, Eds. ACM Press, Oct. 2015, pp. 579-590.

[57] P. Mohassel and M. Franklin, "Efficiency tradeoffs for malicious twoparty computation," in PKC 2006, ser. LNCS, M. Yung, Y. Dodis, A. Kiayias, and T. Malkin, Eds., vol. 3958. Springer, Apr. 2006, pp. $458-473$.

[58] P. Mohassel and B. Riva, "Garbled circuits checking garbled circuits: More efficient and secure two-party computation," in CRYPTO 2013, Part II, ser. LNCS, R. Canetti and J. A. Garay, Eds., vol. 8043 Springer, Aug. 2013, pp. 36-53.

[59] B. Mood, D. Gupta, K. R. B. Butler, and J. Feigenbaum, "Reuse it or lose it: More efficient secure computation through reuse of encrypted values," in ACM CCS 2014, G.-J. Ahn, M. Yung, and N. Li, Eds. ACM Press, Nov. 2014, pp. 582-596.

[60] J. B. Nielsen, P. S. Nordholt, C. Orlandi, and S. S. Burra, "A new approach to practical active-secure two-party computation," in CRYPTO 2012, ser. LNCS, R. Safavi-Naini and R. Canetti, Eds., vol. 7417. Springer, Aug. 2012, pp. 681-700.

[61] J. B. Nielsen and C. Orlandi, "LEGO for two-party secure computation," in TCC 2009, ser. LNCS, O. Reingold, Ed., vol. 5444. Springer, Mar. 2009, pp. 368-386.

[62] T. P. Pedersen, "Non-interactive and information-theoretic secure ver- ifiable secret sharing," in CRYPTO 1991, ser. LNCS, J. Feigenbaum, Ed., vol. 576. Springer, Aug. 1992, pp. 129-140.

[63] C. Peikert, V. Vaikuntanathan, and B. Waters, "A framework for efficient and composable oblivious transfer," in CRYPTO 2008, ser. LNCS, D. Wagner, Ed., vol. 5157. Springer, Aug. 2008, pp. 554-571.

[64] B. Pinkas, T. Schneider, N. P. Smart, and S. C. Williams, "Secure two-party computation is practical," in ASIACRYPT 2009, ser. LNCS, M. Matsui, Ed., vol. 5912. Springer, Dec. 2009, pp. 250-267.

[65] P. Rindal and M. Rosulek, "Faster malicious 2-party secure computation with online/offline dual execution," in USENIX Security 2016. USENIX Association, 2016, pp. 297-314.

[66] a. shelat and C.-H. Shen, "Two-output secure computation with malicious adversaries," in EUROCRYPT 2011, ser. LNCS, K. G. Paterson, Ed., vol. 6632. Springer, May 2011, pp. 386-405.

[67] _ "Fast two-party secure computation with minimal assumptions," in ACM CCS 2013, A.-R. Sadeghi, V. D. Gligor, and M. Yung, Eds. ACM Press, Nov. 2013, pp. 523-534.

[68] N. Smart and S. Tillich. Circuits of Basic Functions Suitable For MPC and FHE. [Online]. Available: http://www.cs.bris.ac.uk/Research/ CryptographySecurity/MPC/

[69] X. Wang, A. J. Malozemoff, and J. Katz, "Faster two-party computation secure against malicious adversaries in the single-execution setting," Cryptology ePrint Archive, Report 2016/762, 2016, http://eprint.iacr. org/2016/762.

[70] A. C.-C. Yao, "Protocols for secure computations (extended abstract)," in FOCS 1982. IEEE Computer Society Press, Nov. 1982, pp. 160164.

[71] - "How to generate and exchange secrets (extended abstract)," in FOCS 1986. IEEE Computer Society Press, Oct. 1986, pp. 162-167.

[72] S. Zahur, M. Rosulek, and D. Evans, "Two halves make a whole reducing data transfer in garbled circuits using half gates," in EUROCRYPT 2015, Part II, ser. LNCS, E. Oswald and M. Fischlin, Eds., vol. 9057. Springer, Apr. 2015, pp. 220-250.

[73] H. Zhou and J. Bruck, "Linear extractors for extracting randomness from noisy sources," in ISIT 2011, A. Kuleshov, V. Blinovsky, and A. Ephremides, Eds. IEEE, 2011, pp. 1738-1742. 